\title{
Article \\ A Modified Ant Lion Optimization Method and Its Application for Instance Reduction Problem in Balanced and Imbalanced Data
}

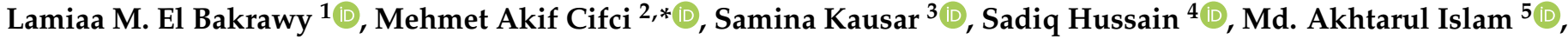 \\ Bilal Alatas ${ }^{6}\left(\mathbb{B}\right.$ and Abeer S. Desuky ${ }^{1}[\mathbb{C}$
}

check for updates

Citation: El Bakrawy, L.M.; Cifci,

M.A.; Kausar, S.; Hussain, S.; Islam,

M.A.; Alatas, B.; Desuky, A.S. A

Modified Ant Lion Optimization

Method and Its Application for

Instance Reduction Problem in

Balanced and Imbalanced Data.

Axioms 2022, 11, 95. https://doi.org/

10.3390/axioms11030095

Academic Editor: Nodari Vakhania

Received: 5 February 2022

Accepted: 22 February 2022

Published: 24 February 2022

Publisher's Note: MDPI stays neutral with regard to jurisdictional claims in published maps and institutional affiliations.

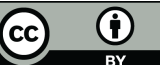

Copyright: (c) 2022 by the authors. Licensee MDPI, Basel, Switzerland. This article is an open access article distributed under the terms and conditions of the Creative Commons Attribution (CC BY) license (https:/ / creativecommons.org/licenses/by/ $4.0 /)$.
1 Mathematics Department, Faculty of Science, Al-Azhar University, Cairo 11754, Egypt; dr_lamiaa_el_bakrawy@azhar.edu.eg (L.M.E.B.); abeerdesuky@azhar.edu.eg (A.S.D.)

2 Department of Computer Engineering, Bandirma Onyedi Eylul University, Balikesir 10200, Turkey

3 Department of Computer Science and Information Technolog, University of Kotli Azad Jammu and Kashmir, Kotli 11100, Pakistan; saminamalik7@yahoo.com

4 Examination Branch, Dibrugarh University, Dibrugarh 786004, India; sadiq@dibru.ac.in

5 Statistics Discipline, Khulna University, Khulna 9208, Bangladesh; akhtarulstat@ku.ac.bd

6 Software Engineering, Firat University, Elazig 23100, Turkey; balatas@firat.edu.tr

* Correspondence: themanoftalent@gmail.com

\begin{abstract}
Instance reduction is a pre-processing step devised to improve the task of classification. Instance reduction algorithms search for a reduced set of instances to mitigate the low computational efficiency and high storage requirements. Hence, finding the optimal subset of instances is of utmost importance. Metaheuristic techniques are used to search for the optimal subset of instances as a potential application. Antlion optimization (ALO) is a recent metaheuristic algorithm that simulates antlion's foraging performance in finding and attacking ants. However, the ALO algorithm suffers from local optima stagnation and slow convergence speed for some optimization problems. In this study, a new modified antlion optimization (MALO) algorithm is recommended to improve the primary ALO performance by adding a new parameter that depends on the step length of each ant while revising the antlion position. Furthermore, the suggested MALO algorithm is adapted to the challenge of instance reduction to obtain better results in terms of many metrics. The results based on twenty-three benchmark functions at 500 iterations and thirteen benchmark functions at 1000 iterations demonstrate that the proposed MALO algorithm escapes the local optima and provides a better convergence rate as compared to the basic ALO algorithm and some well-known and recent optimization algorithms. In addition, the results based on 15 balanced and imbalanced datasets and 18 oversampled imbalanced datasets show that the instance reduction proposed method can statistically outperform the basic ALO algorithm and has strong competitiveness against other comparative algorithms in terms of four performance measures: Accuracy, Balanced Accuracy (BACC), Geometric mean (G-mean), and Area Under the Curve (AUC) in addition to the run time. MALO algorithm results show increment in Accuracy, BACC, G-mean, and AUC rates up to 7\%, $3 \%, 15 \%$, and $9 \%$, respectively, for some datasets over the basic ALO algorithm while keeping less computational time.
\end{abstract}

Keywords: antlion optimization; instance reduction; nature-inspired optimization; machine learning

MSC: 97P10

\section{Introduction}

Machine Learning plays a crucial role in extracting useful information in different research domains, for instance, medical data analysis [1-4], computer vision [5], road accident analysis [6], educational data mining [7], sentiment analysis [8] and many more. Instance reduction is one of the prime pre-processing tasks in machine learning applications. 
Before employing instance-based learning methods, alleviating the noisy, erroneous, and redundant data are highly desirable. Instance reduction mitigates sensitivity to noise and high storage requirements. Eventually, the complication of calculation to understand a superior classification technique decline. There should not be a noteworthy change in between-class distribution after and before data decline. A flawed data reduction technique may eradicate additional instances of one class, resulting in unfair datasets.

Instance reduction can be applied for both balanced and imbalanced data to improve classification performance. Researchers studied the instance decline from class-balanced data. However, there are not many studies on class-imbalanced data. Researchers attract to this issue in recent times because of practical applications in this domain [9]. The imbalanced dataset poses difficulties in learning tasks. Traditional methods are exploited to learn from imbalanced data. Satisfactory outcomes are not yielded as these traditional methods execute excellent coverage for the mainstream class while marginal classes are disregarded. In some cases, high accuracies are demonstrated, but the outcome is not as trustworthy as the cardinality of the majority class is high contrasted to the minority class. For instance, the reduction to keep intact between-class distribution is vital. In imbalanced datasets, the minority class instances are also essential. Such instances may be treated as noise or outliers. However, these minority class instances must not be lessened while applying an instance reduction method. Hence, to take care of that, special techniques are needed.

Among different instance reduction methods for imbalanced data, data-level methods are of utmost importance. These data-level techniques can be classified into two types: oversampling, the size of the marginal class is enhanced and under-sampling, the size of the mainstream class is lessened, ensemble-based methods, and cost-sensitive methods. Various research papers suggested that evolutionary-based techniques performed better than the non-evolutionary ones in imbalanced dataset analysis and instance reduction.

Researchers have gained interest over the years to locate optimal values of the solution variables to meet definite conditions in case of global optimization concerns. Gigantic computational efforts are required for classical optimization methods that are inclined to be unsuccessful as the problem search space escalates. Meta-heuristic algorithms have come into the picture that exhibits better computational efficacy in evading local minima [10]. These meta-heuristic algorithms showcased their superiority for tackling complex issues in different domains for the subsequent causes. (i) They can circumvent local minima; (ii) the gradient of the objective function is not needed; (iii) they are easy to implement and simple; (iv) different problems of various fields can be solved by utilizing it. The increasing processing power of computers has a positive impact on the development of such algorithms.

The metaphors applied in metaheuristic techniques are plants, humans, birds, the ecosystem, water, gravitational forces, and electromagnetic forces. As described in Figure 1, it can be divided into two categories. The first category mimics physical or biological phenomena and three sub-categories come into existence from it. They are swarm-based, physics-based, and evolution-based techniques. Human phenomena are the main inspiration behind the second category.

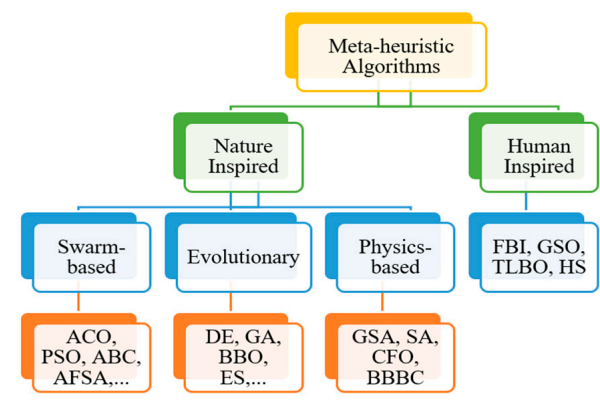

Figure 1. Different categories of meta-heuristic algorithms [10]. 
Abbreviations: ACO: Ant Colony Optimization, PSO: Particle Swarm Optimization, ABC: Artificial Bee Colony, AFSA: Artificial Fish Swarm Algorithm, DE: Differential Evolution, GA: Genetic Algorithm, BBO: Biogeography-Based Optimizer, ES: Evolution Strategy, GSA: Gravitational Search Algorithm, SA: Simulated Annealing, CFO: Central Force Optimization, BBBC: Big-Bang Big-Crunch, FBI: Forensic-Based Investigation, GSO: Group Search Optimizer, TLBO: Teaching-Learning-Based Optimization, HS: Harmony Search.

Metaheuristic techniques were utilized for different real-world applications, Negi et al. [11] proposed a hybrid approach with PSO and GWO methods and dubbed it HPSOGWO to tackle optimization problems and reliability allocation of life support systems and Complex bridge systems. In [12], the authors devised a modified genetic algorithm (MGA) with a novel selection method, namely a generation-dependent mutation and an in-vitrofertilization-based crossover. They applied their technique in commercial ship scheduling and routing with dynamic demand and supply environments. Their model could lessen risks and abate port time with a static load factor. Ganguly in [13] proposed a framework for simultaneous optimization of Distributed Generation (DG) network performance index and penetration level to acquire the optimal sizes, numbers, and sites of DG units. He formulated two objective functions. Network performance index and DG penetration level were the two. Multi-objective particle swarm optimization was utilized by his solution framework and was validated on a distribution system comprising 38 nodes.

In [14], the authors implemented a general type-2 fuzzy classification method for the medical assistance and the optimization of the general type- 2 membership functions parameters using ALO for comparison these two type classifiers with the Framingham dataset. A general type-2 fuzzy classifier had been applied on the Jetson Nano hardware Development Board and execution time of the type- 1 and type- 2 fuzzy classification techniques were compared. A novel metaheuristic method called the Slime Mold Algorithm (SMA) was proposed in [15]. A fuzzy controller tuning technique is also offered and the concept of enhancing the performance of metaheuristics with information feedback approaches has been applied. The fuzzy controllers and their tuning methods were validated in real-time with angular position control of the laboratory servo framework. In [16], a survey was presented on scientific literature works that dealt with Type-2 fuzzy logic controllers devised utilizing nature-inspired optimization techniques. Their review exploited the most widespread optimizers to attain the key parameters on Type- 2 and Type- 1 fuzzy controllers to enhance the gained outcome. The PSO method was integrated in [17], with the Multi-Verse Optimizer (MVO) to classify endometrial carcinoma with gene expression by optimizing the parameters of the Elman neural network.

Swarm intelligence methods were also utilized for feature reduction. Gupta et al. [18] presented a revised antlion optimization procedure to better identify thyroid infection. To mitigate the computational time and enhance the classification accuracy, the proposed method was exploited as a feature reduction technique to detect the vital attributes from a large set of attributes. Their method had successfully eradicated $71.5 \%$ of irrelevant features. Based on Stochastic Fractal Search (SFS), El-Kenawy et al. [19] introduced a Modified Binary GWO (MbGWO) to determine key characteristics by attaining the exploitation and exploration balance. They tested their MbGWO-SFS method with 19 machine learning datasets from the University of California, Irvine (UCI). Comparison with the state-ofthe-art optimization methods demonstrated the superiority of the method. Lin et al. [20] applied modified cat swarm optimization (CSO) that outperformed PSO. The limitation of CSO is that its computation time is long. Hence, their modified version was called ICSO. Their method selected features in big data-related text classification. To propose a feature selection method, Wan et al. [21] utilized a customized binary-coded ant colony optimization (MBACO) method in combination together with a genetic algorithm. Their technique comprised of two models, the pheromone density model (PMBACO) and the visibility density model (VMBACO). The results acquired by GA were applied as early pheromone evidence in the PMBACO model, whereas the solution attained by GA was employed as visibility information in the VMBACO model. Based on the modified grasshopper 
optimization method, Zakeri et al. [22] devised a new feature selection technique. The novel method dubbed Grasshopper Optimization Algorithm for Feature Selection (GOFS) replicated the duplicate features with promising features by applying statistical techniques while performing iterations.

Instance reduction is a pre-processing task devised to improve learning jobs. Nanni et al. [23] developed an effective technique based on particle swarm optimization for prototype reduction. Their technique minimized the error rate on the training set. Zhai et al. [24] introduced a novel immune binary particle swarm optimization technique for time series classification, which searched for the smallest instance combination with the highest classification accuracy. Hamidzadeh et al. [25] presented a Large Margin Instance Reduction Algorithm (LMIRA) that kept border instances and removed the non-border ones. The algorithm considered the instance reduction issue as a constrained binary optimization problem, and a filled function algorithm was exploited to tackle the issue. The reduction process's basic was relied on the hyperplane that separated the two-class data and demonstrated large margin separation. Saidi et al. [26] proposed a novel instance selection method dubbed Ensemble Margin Instance Selection (EMIS). The ensemble margin was employed in their method. They applied their method for automatic recognition and selection of white blood cells WBC in cytological images.

Carbonera and Abel [27] devised an effective and simple density-based framework for instance collection termed local density-based instance selection (LDIS). Their technique kept the densest instances in an arbitrary neighborhood by examining the instances of each class. For evaluating the accuracy, they applied the K-Nearest Neighbor (KNN) algorithm. de Haro-García et al. [28] utilized boosting method to obtain reduced instances to achieve better accuracy. The stepwise addition of instances was performed by applying the weighting of instances from the building of ensembles of classifiers.

Numerous modified versions of Antlion optimizers have been proposed for solving different research problems. In [29], Wang et al. proposed an enhanced alternate method for Antlion Optimizer (ALO), incorporating opposition-based training with two functional operators centered on differential evolution, named MALO, which is suggested to deal with the implicit vulnerabilities of conventional ALO. Pierezan et al. [30] suggested four multi-objective ALO (MOALO) methods utilizing swarming distance, supremacy idea for choosing the elite, and tournament collection techniques with various programs to pick the chief. Assiri et al. [31] showed the benefits and different categories like Modified, Hybrid, and Multi-Objective of ALO algorithms after giving a detailed introduction of this procedure. This paper also discussed the applications and foundations of this method and finished with some suggestions and possible directions in the future.

In the literature, different metaheuristic and optimization algorithms were proposed to enhance the performance of classification using the instance reduction issue. However, as far as the authors are aware, this is the first time ALO algorithm or a modified version of it is proposed to solve the instance reduction issue in balanced and imbalanced data. In this paper, MALO will be utilized to enhance the ALO's ability to escape the local optima while providing a better convergence rate and enhancing the classification performance in real-world datasets by optimized instance reduction.

The main contributions of this work are summarized as follows:

(1) Since the ALO algorithm suffers from local optima stagnation and slow convergence speed for some optimization problems [32], this study's intention is to propose a new modified antlion optimization (MALO) algorithm to enhance the optimization efficiency and accuracy of ALO by adding a new parameter depends on the step length of each ant while updating the antlion position based on the parameter, upper and lower bounds of search space.

(2) The proposed MALO algorithm is tested on twenty-three benchmark functions at 500 iterations and thirteen benchmark functions at 1000 iterations. The results provide evidence that the suggested MALO escapes the local optima and provides a better 
convergence rate as compared to the basic ALO algorithm, some well-known and recent optimization algorithms.

(3) Furthermore, 15 balanced and imbalanced datasets were employed to test the performance of the proposed MALO algorithm on reducing instances of the training data and the results are compared with some well-known optimization algorithms.

(4) The Wilcoxon signed-rank test is also applied. The results showcase that the proposed instance reduction method statistically outperforms the MALO algorithm and other comparable optimization techniques based on the recorded Accuracy, BACC, G-Mean, and AUC metrics while keeping less computational time.

(5) Moreover, antlion optimization and MALO were used to perform training data reduction for 18 oversampled imbalanced datasets, and learning is performed using Support Vector Machines (SVM) classifier in all performed experiments. The results are compared with one novel resampling method and two recent algorithms.

\section{Methodology}

\subsection{Antlion Optimizer}

Seyedali Mirjalili proposed an Antlion Optimizer (ALO) in 2015 utilizing the ant and lions hunting process [33]. This method consists of five major steps of hunting, specifically random walk of agents, entrapment of ants in the trap, constructing traps, reconstructing traps, and capturing prey. Ant and antlion interactions are followed in the ALO algorithm where the ants are chased by antlions utilizing the traps, and ants are authorized to drift into the search area stochastically for food.

The following matrices represent the matrices for representing the place of $p$ ants and $p$ antlions, where $q$ is the number of variables (dimension).

$$
S_{A n t}=\left[\begin{array}{cccc}
A n t_{1,1} & A n t_{1,2} & \ldots & A n t_{1, q} \\
A n t_{2,1} & A n t_{2,2} & \ldots & A n t_{2, q} \\
\ldots & \ldots & \ldots & \ldots \\
A n t_{p, 1} & A n t_{p, 2} & \ldots & A n t_{p, q}
\end{array}\right]
$$

and

$$
S_{\text {Antlion }}=\left[\begin{array}{cccc}
\text { Antlion }_{1,1} & \text { Antlion }_{1,2} & \ldots & \text { Antlion }_{1, q} \\
\text { Antlion }_{2,1} & \text { Antlion }_{2,2} & \ldots & \text { Antlion }_{2, q} \\
\ldots & \ldots & \ldots & \ldots \\
\text { Antlion }_{p, 1} & \text { Antlion }_{p, 2} & \ldots & \text { Antlion }_{p, q}
\end{array}\right]
$$

If $f$ indicated the fitness function for the duration of optimization, then the matrices developed the matrices for savings the fitness value (objective) of $p$ ants $\left(S_{\text {OAnt }}\right)$ and $p$ antlions $\left(S_{\text {OAntlion }}\right)$.

$$
S_{O A n t}=\left[\begin{array}{c}
f\left(\left[\text { Ant }_{11}, A n t_{12}, \ldots, A n t_{1 q}\right]\right) \\
f\left(\left[\text { Ant }_{21}, A n t_{22}, \ldots, A n t_{2 q}\right]\right) \\
\ldots \\
f\left(\left[A n t_{p 1}, A n t_{p 2}, \ldots, A n t_{p q}\right]\right)
\end{array}\right]
$$

and

$$
S_{\text {OAntlion }}=\left[\begin{array}{c}
f\left(\left[\text { Antlion }_{11}, \text { Antlion }_{\mathrm{L}_{12}}, \ldots, \text { Antlion }_{1 q}\right]\right) \\
f\left(\left[\text { Antlion }_{21}, \text { Antlion }_{22}, \ldots, \text { Antlion }_{2 q}\right]\right) \\
\ldots \\
f\left(\left[\text { Antlion }_{p 1}, \text { Antlion }_{p 2}, \ldots, \text { Antlion }_{p q}\right]\right)
\end{array}\right]
$$

ALO algorithm contains six operators.

(i) Random Walks of Ants. At every single stage of optimization, ants revise their places with a random walk $X(t)$. A random walk is calculated according to Equation (5). Where the cumulative sum is computed by cumsum, the maximum number of iterations 
is $T$, the present repetition (iteration) is indicated by $t$ and rand implies a random number using the uniform probability distribution with the range [0,1].

$$
X(t)=\left[0, \operatorname{cumsum}\left(2 r\left(t_{1}\right)-1\right), \operatorname{cumsum}\left(2 r\left(t_{2}\right)-1\right), \ldots, \operatorname{cumsum}\left(2 r\left(t_{T}\right)-1\right)\right]
$$

where the statistic function $r(t)$ is illustrated as in Equation (6).

$$
r(t)=\left\{\begin{array}{c}
1 \text { if rand }>0.5 \\
0 \text { if rand } \leq 0.5
\end{array}\right.
$$

The ants are normalized as each search area has a province to maintain the random walks within the search area. To normalize the process the subsequent equation (min-max normalization), Equation (7) is used before updating the position of ants. Where the lowest of random walk of the $i$ th variable is $a_{i}, q_{i}$ is the highest of random walk in the $i$ th variable, $c_{i}^{t}$ implies the smallest of the $i$ th variable at the $t$ th repetition, $q_{i}^{t}$ implies the highest of the $i$ th variable at the $t$ th repetition.

$$
X_{i}^{t}=\frac{\left(X_{i}^{t}-a_{i}\right) \times\left(q_{i}-c_{i}^{t}\right)}{\left(q_{i}^{t}-a_{i}\right)}+c_{i}^{t}
$$

(ii) Trapping in Antlion's Pits. The traps of Antlions affect the random walks of ants. Equations (8) and (9) illustrate that ant's random walk in a hypersphere is indicated by the $c$ and $q$ vectors across a chosen antlion.

In these equations, $l c^{t}$ implies the lowest among total variables at the $t$ th repetition, the vector $h q^{t}$ suggests involving the vector of the highest of total variables at the th repetition, $c_{i}^{t}$ implies the lowest of total variables for the $i$ th ant, $q_{i}^{t}$ indicates the highest of total variables for the $i$ th ant, and Antlion $n_{j}^{t}$ indicates a place of chosen $j$ th antlion of $t$ th repetition. Where $i$ is the index of the current ant and $j$ is the index of the current antlion.

$$
c_{i}^{t}=\text { Antlion }_{j}^{t}+l c^{t}
$$

and

$$
q_{i}^{t}=\text { Antlion }_{j}^{t}+h q^{t}
$$

(iii) Building Trap. To choose the fitter antlions intended for capturing ants founded on the fitness value for the period of optimization, a roulette wheel in the ALO procedure is utilized.

(iv) Sliding Ants towards Antlion. Antlions can develop traps proportionately and ants change arbitrarily corresponding to the fitness values. Antlions shoot sands outwards when the ants are in the trap in the middle of the pit. This performance slides down the stuck ant, which is attempting to avoid the trap; in this circumstance, the range of the ants' random walks hypersphere is reduced adjustably. Considering $r c^{t}$ and $r q^{t}$ as the reduced vectors of the lowest and highest random walks among total variables at the $t$ th repetition, respectively:

$$
r c^{t}=\frac{l c^{t}}{I}
$$

and

$$
r q^{t}=\frac{h q^{t}}{I}
$$

where $l c^{t}$ implies the lowest among total variables at the $t$ th repetition, the vector $h q^{t}$ suggests involving the vector of the highest of total variables at the $t$ th repetition, and $I$ indicate a ratio that calculated as:

$$
I=10^{w}(t / T)
$$


where, $w$ implies a fixed value indicated centered on the present reptation $t[((w=2$, if $t>0.1 \mathrm{~T}),(w=3$ if $t>0.5 \mathrm{~T}),(w=4$ if $t>0.75 \mathrm{~T}),(w=5$ if $t>0.9 \mathrm{~T})$ and $(w=6$ if $t>0.95 \mathrm{~T})$ ]. Mostly, the fixed value $w$ is able to accommodate the intensity of manipulation and precision.

Equations (10) and (11) decrease the range of the revising ants' places and simulate the sliding method of an ant inside the pits.

(v) Catching Prey and Rebuilding the Pit. The ant gets caught by the antlion as soon as it goes to the underside of the pit. The antlion revises the place to the most recent situation of the tracked ant to enhance its probability of capturing new-found prey as in Equation (12):

$$
\text { Antlion }{ }_{j}^{t}=\text { Ant }_{i}^{t} \text { iff }\left(\text { Antlion }_{j}^{t}\right)<f\left(A n t_{i}^{t}\right)
$$

where $A n t l i o n_{j}^{t}$ indicated the place of the chosen $j$ th antlion at the $t$ th repetition and $A n t_{i}^{t}$ implies a place of the $i$ th ant at the thepetition.

(vi) Elitism. The essential feature of the evolutionary processes to preserve the finest explanation(s) achieved at every phase of the optimization procedure is elitism. The greatest antlion is an elite in the ALO procedure. Every single ant arbitrarily walks all over a carefully chosen antlion via the roulette wheel and the elite instantaneously as defined in Equation (13).

$$
A n t_{i}^{t}=\frac{R_{A}^{t}+R_{E}^{t}}{2}
$$

In this equation, the random walk indicated by $R_{A}^{t}$ all over the antlion is chosen by the roulette wheel at the $t$ th repetition, the random walk all over the elite is implied by $R_{E}^{t}$ at the $t$ th repetition.

Let a function that produces the arbitrary primary results be $X, Y$ directs the preliminary population presented by the function $X$, and $Z$ comes back true when the ending principle is assured. Utilizing the above-recommended operations, the ALO procedure is identified as a three-tuple which can be shown as follows:

$$
A L O(X, Y, Z)
$$

where the functions $X, Y$, and $Z$ are defined as follows

$$
\begin{gathered}
\varnothing \stackrel{X}{\rightarrow}\left\{S_{\text {Ant }}, S_{\text {OAnt }}, S_{\text {Antlion }}, S_{\text {OAntlion }}\right\} \\
\left\{S_{\text {Ant }}, S_{\text {Antlion }}\right\} \stackrel{Y}{\rightarrow}\left\{S_{\text {Ant }}, S_{\text {Antlion }}\right\} \\
\left\{S_{\text {Ant }}, S_{\text {Antlion }}\right\} \stackrel{Z}{\rightarrow}\{\text { true }, \text { false }\}
\end{gathered}
$$

Here, $S_{\text {Ant }}$ implies the matrix of the place of the ants, $S_{\text {Antlion }}$ incorporates antlions' place, $S_{O A n t}$ includes the ants' consequent fitness, and $S_{O A n t l i o n}$ has antlions' fitness.

\subsection{Modified Antlion Optimization (Malo) Method and Its Adaption for Instance Reduction}

The ALO algorithm updates the ants' positions based on random walks across the antlion which is elected by the roulette wheel and the elite. Then, by updating the elite during the method of searching, the fittest one is chosen. However, it suffers from local optima stagnation and slow convergence speed for some optimization problems [32]. MALO is proposed to enhance the optimization precision and effectiveness of ALO by adding a new parameter $T u$ which depends on the step length of each ant while updating the antlion position based on the parameter $T u$, upper and lower bounds of search space. The pseudocode of the MALO optimization method is presented in Algorithm 1.

For validating the MALO performance, it is tested using benchmark functions and applied for instance reduction on many real-world datasets. 


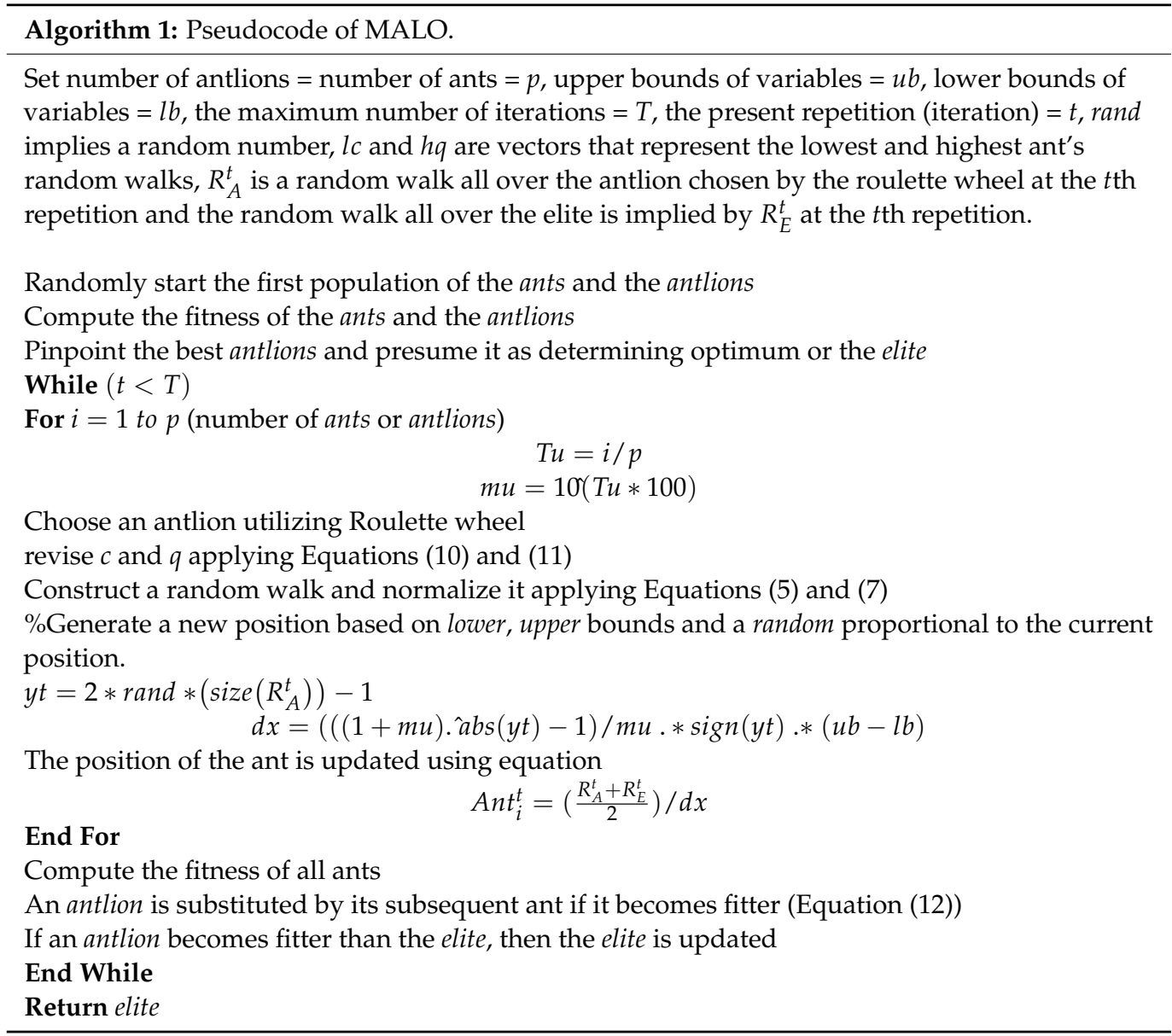

The test is performed on benchmark functions to prove that the proposed MALO algorithm has the ability to escape the local optima and provides a better convergence rate compared to the basic ALO algorithm and some other well-known optimizers. This test is applied on two cases: Case I is performed on twenty-three benchmark functions and results are recorded at 500 iterations. Case II is performed on thirteen benchmark functions and the results are recorded at 1000 iterations.

The application of MALO for the instance reduction problem is then performed in two scenarios: the first scenario is applying MALO to reduce the instances of a training set of both balanced and imbalanced datasets in their original form and the second scenario is performed on the oversampled imbalanced datasets. In the first scenario, the proposed MALO starts the search with randomly generated search agents (antlions) for instance reduction. The binary encoding type is used for the representation scheme of the proposed MALO in the instance reduction problem. In this encoding type, each search agent is represented as a vector of binary elements, and the data instances are treated as either present ' 1 ' or absent ' 0 '. The $1 \mathrm{~s}$ represent the remained instances while $0 \mathrm{~s}$ represent the removed instances. The search agents in MALO are evaluated by G-mean, which is defined in Equation (21), as fitness value. The G-mean is used as an accuracy metric for imbalanced data because it simultaneously can measure the accuracies of both classes (majority and minority).

Figure 2 depicts the flowchart of the MALO method and its implication in the instance decline challenge for the balanced and imbalanced real-world datasets. As shown in this figure, firstly, the original data (balanced or imbalanced) is divided into three subsets as training $(50 \%)$, validation $(25 \%)$, and testing $(25 \%)$, consequently. The proposed MALO tries to find an optimal subset from the training set, tested in each iteration using the validation set and G-mean of the SVM classifier as a fitness function. The population used is vectors of zeros and ones; each vector is the same size as the training set examples. One 
denotes the corresponding example stay in the training set, and zero implies removing the related example from the set. The SVM classifier is trained using the training subset corresponding for each vector of population and evaluated using the validation set. MALO uses G-mean as its fitness function in each iteration till finding the optimal training set. When the termination criteria are satisfied, the best search agent (antlion) is assumed as converged. This search agent with the highest fitness value is decoded for the solution that consists of an optimal reduced training dataset. The SVM classifier is trained using the optimal training subset resulting from the MALO and tested using the testing subset.

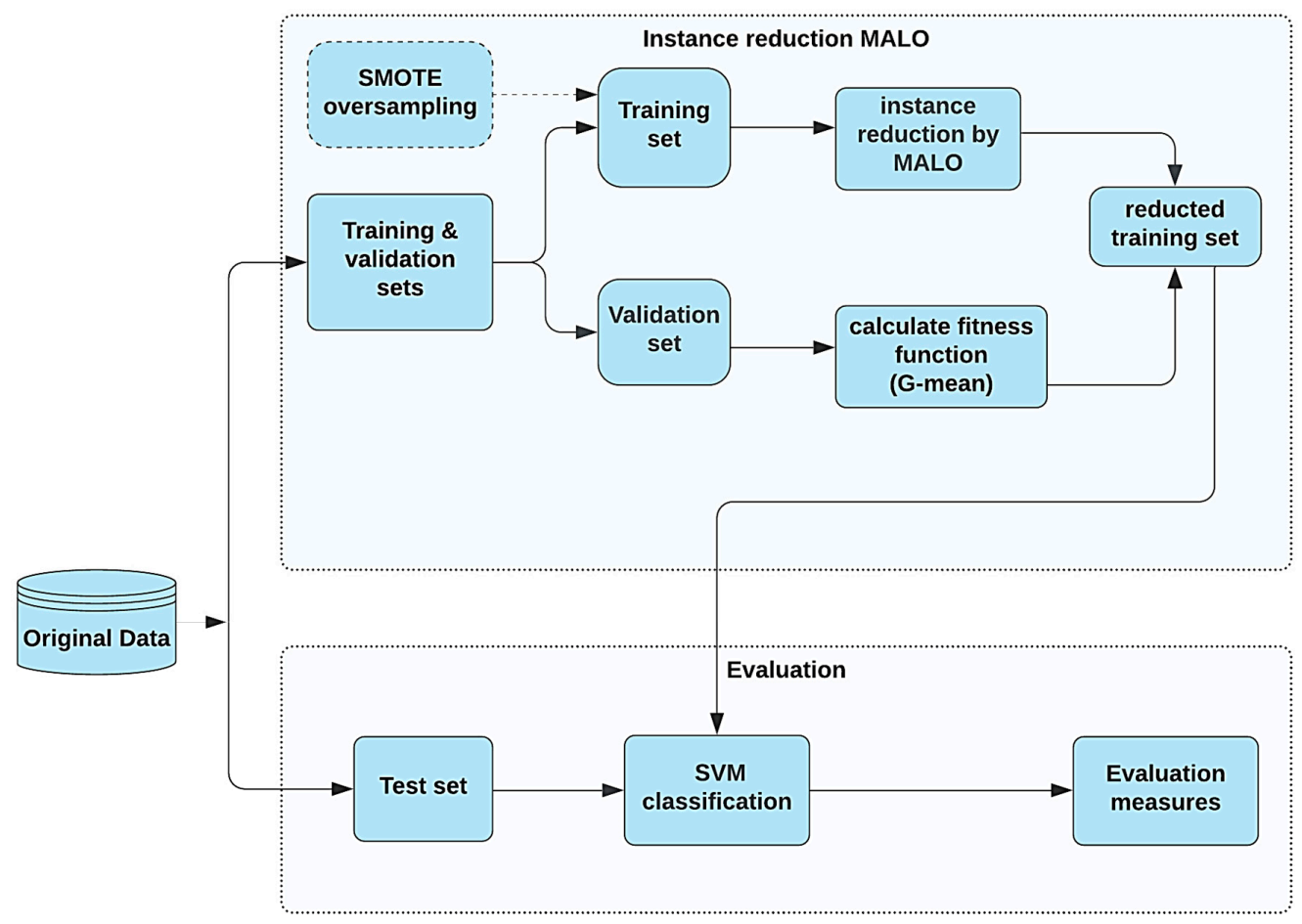

Figure 2. Flow chart of MALO method.

Figure 2 also indicates the second scenario, which is performed on the oversampled imbalanced datasets. After dividing the dataset into training, validation, and testing subsets, the imbalanced training set is rebalanced by a specific oversampling algorithm Synthetic Minority Oversampling Technique (SMOTE) [34]; subsequently, the proposed MALO tries to find an optimal subset from the obtained balanced dataset.

State-of-the-art oversampling algorithms can be fully used to obtain an ideal training set. The MALO can reduce the number of instances of both majority and minority classes and provides a training set that is more suitable for a specified classifier.

To evaluate the accomplishment of the MALO instance reduction technique experiments were performed and the results were compared with state-of-the-art instance reduction methods: Grey Wolf Optimization (GWO) [35], Whale Optimization Algorithm (WOA) [36], and one recently published resampling method.

A Support Vector Machine (SVM) [37] was used in our experiments to measure the classification performance of the reduced training data resulting from the MALO instance reduction algorithm using numerous evaluation metrics as defined in Equations (18)-(22).

For evaluation metrics, let $T N$ represents true negatives and TP represents true positives, FN implies false negatives, FP denotes false positives, TPR is the true positive rate and TNR is the true negative rate [9].

$$
\text { Accuracy }=\frac{T P+T N}{T P+T N+F P+F N}
$$




$$
\begin{gathered}
\text { Recall }=\text { Sensitivity }=T P R=\frac{T P}{T P+F N} \\
\text { Specificity }=T N R=\frac{T N}{T N+F P} \\
G-\text { mean }=\sqrt{T P R \times T N R} \\
\text { Balanced Accuracy }(B A C C)=(((T P /(T P+F N)+(T N /(T N+F P))) / 2
\end{gathered}
$$

The area under the ROC (Receiver Operating Characteristics) curve (AUC) is a performance measurement for the classification problems at numerous threshold situations. ROC is a likelihood curve and $A U C$ is used to measure or degree of separability.

\section{Results and Discussion}

To prove the efficiency of the suggested MALO algorithm, we tested its performance in two types of experiments: Experiment 1 was performed using benchmark functions, and experiment 2 was conducted using balanced and imbalanced real-world datasets given below.

\subsection{Experiment 1: Results of Malo on Benchmark Functions}

Generally, the benchmark functions can be classified into three types: unimodal functions (Table 1), multimodal functions (Table 2), and fixed-dimension multimodal functions (Table 3). In these tables, Dim represents the dimension of variables, Range denotes the range of variation of optimization variables and $f_{\min }$ represents the optimal value quoted in the literature. Figure 3 shows the $2 \mathrm{D}$ versions of the cost function for $F_{1}, F_{8}$, $F_{15}$, and $F_{22}$ test problems used in this study.

\begin{tabular}{|c|c|c|c|}
\hline Function & Dim & Range & $f_{\min }$ \\
\hline$F_{8}(x)=\sum_{i=1}^{n}-x_{i} \sin \left(\sqrt{\left|x_{i}\right|}\right)$ & 30 & {$[-500,500]$} & $-418.9829 \times 5$ \\
\hline$F_{9}(x)=\sum_{i=1}^{n}\left[x_{i}^{2}-\cos \left(2 \pi x_{i}\right)+10\right]$ & 30 & {$[-5.12,5.12]$} & 0 \\
\hline$F_{10}(x)=-20 \exp \left(-0.2 \sqrt{\frac{1}{n} \sum_{i=1}^{n} x_{i}^{2}}\right)-\exp \left(\frac{1}{n} \sum_{i=1}^{n} \cos \left(2 \pi x_{i}\right)\right)+20+e$ & 30 & {$[-32,32]$} & 0 \\
\hline$F_{11}(x)=\frac{1}{4000} \sum_{i=1}^{n} x_{i}^{2}-\pi_{i=1}^{n} \cos \left(\frac{x_{i}}{\sqrt{i}}\right)+1$ & 30 & {$[-600,600]$} & 0 \\
\hline $\begin{array}{c}F_{12}(x)=\frac{\pi}{n}\left\{10 \sin \left(\pi y_{1}\right)+\sum_{i=1}^{n}\left(y_{i}-1\right)^{2}\left[1+10 \sin ^{2}\left(\pi y_{i+!}\right)\right]+\left(y_{n}-1\right)^{2}\right\} \quad+\sum_{i=1}^{n} u\left(x_{i}, 10,100,4\right) \\
y_{i}=1+\frac{x_{i}+1}{4} u\left(x_{i}, a, k, m\right)=\left\{\begin{array}{c}k\left(x_{i}-a\right)^{m} x_{i}>a \\
0-a<x_{i}<a \\
k\left(-x_{i}-a\right)^{m} x_{i}<-a\end{array}\right.\end{array}$ & 30 & {$[-50,50]$} & 0 \\
\hline$F_{13}(x)=0.1\left\{\sin ^{2}\left(3 \pi x_{1}\right)+\sum_{i=1}^{n}\left(x_{i}-1\right)^{2}\left[1+\sin ^{2}\left(3 \pi x_{i}+1\right)\right]+\left(x_{n}-1\right)^{2}\left[1+\sin ^{2}\left(2 \pi x_{n}\right)\right]\right\}+$ & 30 & {$[-50,50]$} & 0 \\
\hline
\end{tabular}

Table 1. Description of unimodal benchmark functions $\left(F_{1}-F_{7}\right)$.

\begin{tabular}{cccc}
\hline Function & Dim & Range & $f_{\text {min }}$ \\
\hline$F_{1}(x)=\sum_{i=1}^{n} x_{i}^{2}$ & 30 & {$[-100,100]$} & 0 \\
$F_{2}(x)=\sum_{i=1}^{n}\left|x_{i}\right|+\prod_{i=1}^{n}\left|x_{i}\right|$ & 30 & {$[-10,10]$} & 0 \\
$F_{3}(x)=\sum_{i=1}^{n}\left(\sum_{j-1}^{i} x_{j}\right)^{2}$ & 30 & {$[-100,100]$} & 0 \\
$F_{4}(x)=\max _{i}\left\{\left|x_{i}\right|, 1 \leq i \leq n\right\}$ & 30 & {$[-100,100]$} & 0 \\
$F_{5}(x)=\sum_{i=1}^{n-1}\left[100\left(x_{i+1}-x_{i}^{2}\right)^{2}+\left(x_{i}-1\right)^{2}\right]$ & 30 & {$[-30,30]$} & 0 \\
$F_{6}(x)=\sum_{i=1}^{n}\left(\left[x_{i}+0.5\right]\right)^{2}$ & 30 & {$[-100,100]$} & 0 \\
$F_{7}(x)=\sum_{i=1}^{n} i x_{i}^{4}+\operatorname{rand}(0,1)$ & 30 & {$[-1.28,1.28]$} & 0 \\
\hline
\end{tabular}

Table 2. Description of multimodal benchmark functions $\left(F_{8}-F_{13}\right)$. 
Table 3. Description of fixed-dimension multimodal benchmark functions $\left(F_{14}-F_{23}\right)$.

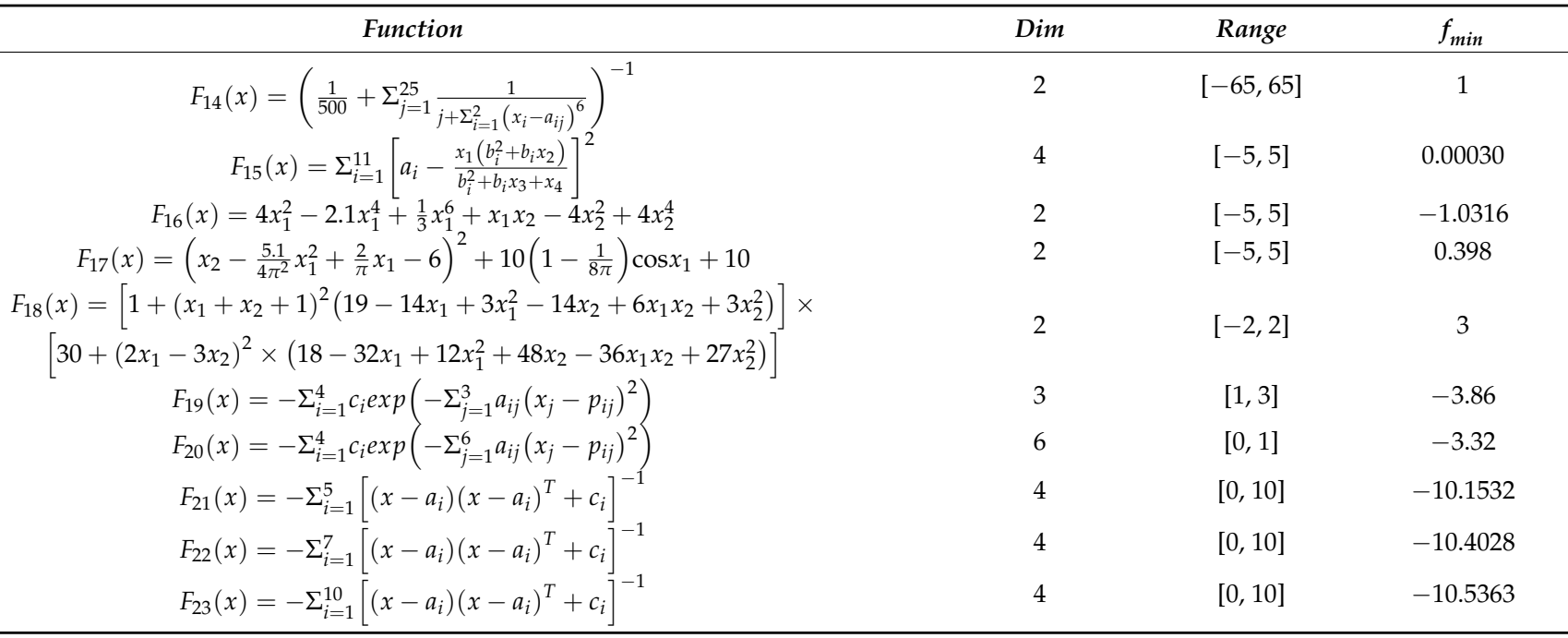
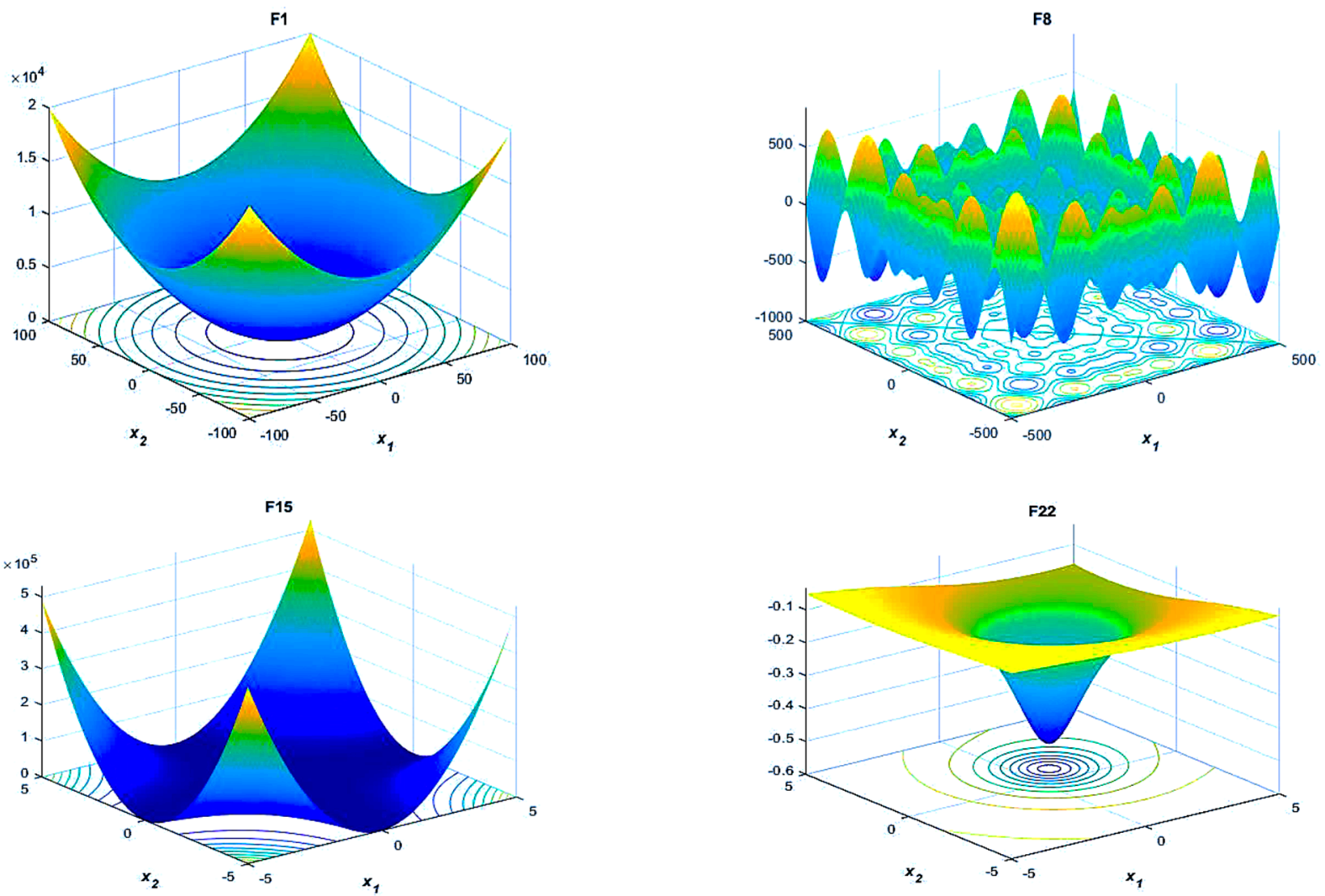

Figure 3. 2-D versions of the cost function for some benchmark problems.

In this paper, the experiments were performed on a 64-bit Windows 10 system using $2.40 \mathrm{GHz}$ frequency, $16 \mathrm{~GB}$ of RAM, Intel(R) Core(TM) i7, and Matlab R2018a. The proposed MALO algorithm was run 30 times independently on each benchmark function using 30 candidate solutions (antlions). The average (AV) and standard deviation (STD) of the best-obtained solution in the last iteration were recorded. To analyze the impact of the maximum number of iterations on the MALO algorithm, experiments were conducted in two cases: Table 4 indicates the comparison for the maximum number of iterations $=500$ (case I), and Tables 5 and 6 for the maximum number of iterations $=1000$ (case II). 
Table 4. Results of malo and other algorithms for benchmark functions (500 iterations).

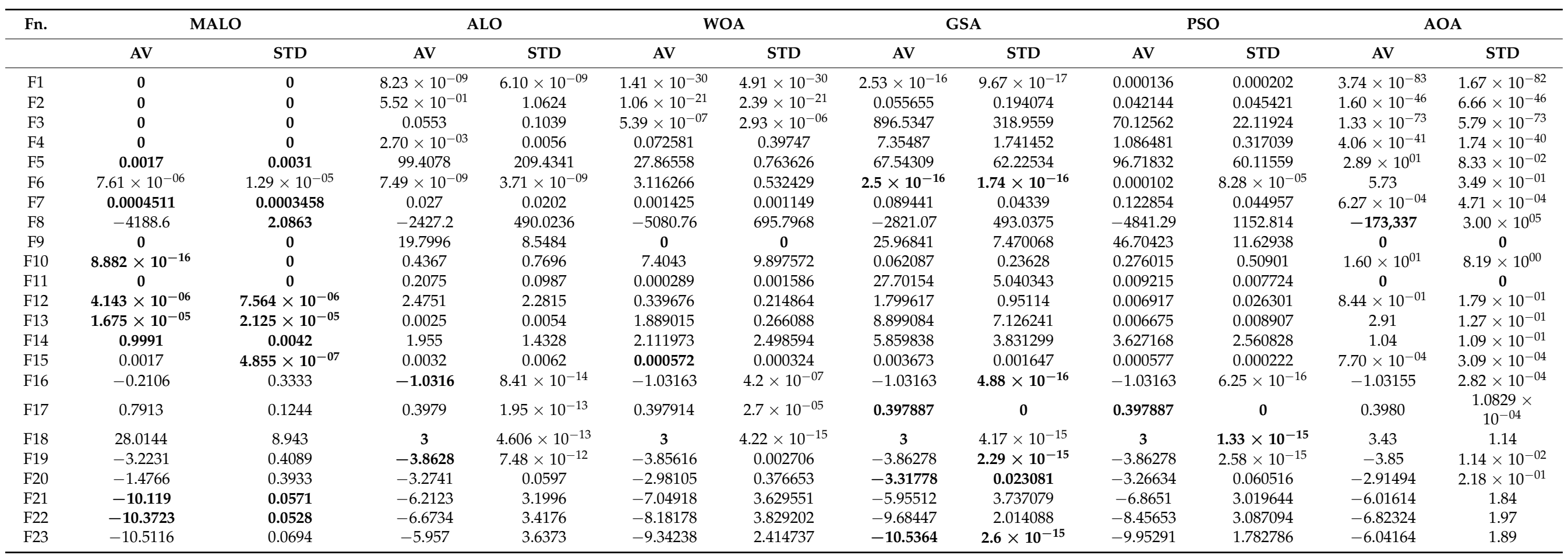


Table 5. Results of malo and other algorithms for unimodal functions (1000 iterations).

\begin{tabular}{|c|c|c|c|c|c|c|c|c|c|c|}
\hline \multirow[t]{2}{*}{ Function } & \multicolumn{2}{|c|}{ MALO } & \multicolumn{2}{|c|}{ ALO } & \multicolumn{2}{|c|}{ BA } & \multicolumn{2}{|c|}{ SMS } & \multicolumn{2}{|c|}{ PSO } \\
\hline & AV & STD & AV & STD & AV & STD & AV & STD & AV & STD \\
\hline F1 & 0 & 0 & $2.59 \times 10^{-10}$ & $1.65 \times 10^{-10}$ & 0.773622 & 0.528134 & 0.056987 & 0.014689 & $2.70 \times 10^{-09}$ & $1.00 \times 10^{-09}$ \\
\hline F2 & 0 & 0 & $1.84 \times 10^{-06}$ & $6.58 \times 10^{-07}$ & 0.334583 & 3.816022 & 0.006848 & 0.001577 & $7.15 \times 10^{-05}$ & $2.26 \times 10^{-05}$ \\
\hline F3 & 0 & 0 & $6.07 \times 10^{-10}$ & $6.34 \times 10^{-10}$ & 0.115303 & 0.766036 & 0.959865 & 0.82345 & $4.71 \times 10^{-06}$ & $1.49 \times 10^{-06}$ \\
\hline F4 & $\mathbf{0}$ & 0 & $1.36 \times 10^{-08}$ & $1.81 \times 10^{-09}$ & 0.192185 & 0.890266 & 0.276594 & 0.005738 & $3.25 \times 10^{-07}$ & $1.02 \times 10^{-08}$ \\
\hline F5 & 0.0005309 & 0.000898 & 0.346772 & 0.109584 & 0.334077 & 0.300037 & 0.085348 & 0.140149 & 0.123401 & 0.216251 \\
\hline F6 & $1.90 \times 10^{-06}$ & $3.31 \times 10^{-06}$ & $2.56 \times 10^{-10}$ & $1.09 \times 10^{-10}$ & 0.778849 & 0.67392 & 0.125323 & 0.084998 & $5.23 \times 10^{-07}$ & $2.74 \times 10^{-06}$ \\
\hline \multirow{2}{*}{ F7 } & $1.83 \times 10^{-04}$ & $1.65 \times 10^{-04}$ & 0.004292 & 0.005089 & 0.137483 & 0.112671 & 0.000304 & 0.000258 & 0.001398 & 0.001269 \\
\hline & \multicolumn{2}{|c|}{ FPA } & \multicolumn{2}{|c|}{ GA } & \multicolumn{2}{|c|}{ FA } & \multicolumn{2}{|c|}{ CS } & \multicolumn{2}{|c|}{ AOA } \\
\hline F1 & $1.06 \times 10^{-07}$ & $1.27 \times 10-07$ & 0.118842 & 0.125606 & 0.039615 & 0.01449 & 0.0065 & 0.000205 & $1.2 \times 10^{-222}$ & 0 \\
\hline F2 & 0.0006242 & 0.000176 & 0.145224 & 0.053227 & 0.050346 & 0.012348 & 0.212 & 0.0398 & $3.5 \times 10^{-122}$ & $7.9 \times 10^{-122}$ \\
\hline F3 & $5.67 \times 10^{-08}$ & $3.90 \times 10^{-08}$ & 0.13902 & 0.121161 & 0.049273 & 0.019409 & 0.247 & 0.0214 & $3 \times 10^{-196}$ & 0 \\
\hline $\mathrm{F} 4$ & 0.0038379 & 0.002186 & 0.157951 & 0.862029 & 0.145513 & 0.031171 & $1.12 \times 10^{-05}$ & $8.25 \times 10^{-06}$ & $2.7 \times 10^{-106}$ & $5.9 \times 10^{-106}$ \\
\hline F5 & 0.7812 & 0.366891 & 0.714157 & 0.972711 & 2.175892 & 1.447251 & 0.007197 & 0.007222 & 8.7651 & 0.1116 \\
\hline F6 & $1.08 \times 10^{-07}$ & $1.25 \times 10^{-07}$ & 0.167918 & 0.868638 & 0.05873 & 0.014477 & $5.95 \times 10^{-05}$ & $1.08 \times 10^{-06}$ & 0.9464 & 0.1763 \\
\hline F7 & 0.0031053 & 0.001367 & 0.010073 & 0.003263 & 0.000853 & 0.000504 & 0.001321 & 0.000728 & 0.000534 & 0.000218 \\
\hline
\end{tabular}

Table 6. Results of malo and other algorithms for multimodal functions (1000 iterations).

\begin{tabular}{|c|c|c|c|c|c|c|c|c|c|c|}
\hline \multirow[t]{2}{*}{ Function } & \multicolumn{2}{|c|}{ MALO } & \multicolumn{2}{|c|}{ ALO } & \multicolumn{2}{|c|}{ BA } & \multicolumn{2}{|c|}{ SMS } & \multicolumn{2}{|c|}{ PSO } \\
\hline & AV & STD & $\mathbf{A V}$ & STD & $\mathrm{AV}$ & STD & AV & STD & AV & STD \\
\hline F8 & -4189.4 & 0.6265 & -1606.28 & 314.4302 & -1065.88 & 858.498 & -4.20735 & $9.36 \times 10^{-16}$ & -1367.01 & 146.4089 \\
\hline F9 & 0 & 0 & $7.71 \times 10^{-06}$ & $8.45 \times 10^{-06}$ & 1.233748 & 0.686447 & 1.32512 & 0.326239 & 0.278588 & 0.218991 \\
\hline F10 & $8.88 \times 10^{-16}$ & 0 & $3.73 \times 10^{-15}$ & $1.50 \times 10^{-15}$ & 0.129359 & 0.043251 & $8.88 \times 10^{-06}$ & $8.56 \times 10^{-09}$ & $1.11 \times 10^{-09}$ & $2.39 \times 10^{-11}$ \\
\hline F11 & 0 & 0 & 0.018604 & 0.009545 & 1.451575 & 0.570309 & 0.70609 & 0.907954 & 0.273674 & 0.204348 \\
\hline F12 & $1.781 \times 10^{-06}$ & $2.90 \times 10^{-06}$ & $9.75 \times 10^{-12}$ & $9.33 \times 10^{-12}$ & 0.395977 & 0.993325 & 0.12334 & 0.040898 & $9.42 \times 10^{-09}$ & $2.31 \times 10^{-10}$ \\
\hline \multirow[t]{3}{*}{ F13 } & $3.78 \times 10^{-06}$ & $5.06 \times 10^{-06}$ & $2.00 \times 10^{-11}$ & $1.13 \times 10^{-11}$ & 0.386631 & 0.121986 & 0.0135 & 0.000288 & $1.35 \times 10^{-07}$ & $2.88 \times 10^{-08}$ \\
\hline & \multicolumn{2}{|c|}{ FPA } & \multicolumn{2}{|c|}{ GA } & \multicolumn{2}{|c|}{ FA } & \multicolumn{2}{|c|}{ CS } & \multicolumn{2}{|c|}{ AOA } \\
\hline & $\mathrm{AV}$ & STD & AV & STD & AV & STD & AV & STD & AV & STD \\
\hline F9 & 0.2732946 & 0.068583 & 0.659271 & 0.815751 & 0.263458 & 0.182824 & 0.127328 & 0.002655 & 10.4661 & 10.068 \\
\hline F10 & 0.0073987 & 0.007096 & 0.956111 & 0.807701 & 0.168306 & 0.050796 & $8.16 \times 10^{-09}$ & $1.63 \times 10^{-08}$ & $8.88 \times 10^{-16}$ & 0 \\
\hline F11 & 0.0850217 & 0.040046 & 0.487809 & 0.217782 & 0.099815 & 0.024466 & 0.122678 & 0.049673 & 0 & 0 \\
\hline F12 & 0.0002657 & 0.000553 & 0.110769 & 0.002152 & 0.126076 & 0.263201 & $5.60 \times 10^{-09}$ & $1.58 \times 10^{-10}$ & 0.6804 & 0.496 \\
\hline F13 & $3.67 \times 10^{-06}$ & $3.51 \times 10^{-06}$ & $1.29 \times 10^{-01}$ & 0.068851 & 0.00213 & 0.001238 & $4.88 \times 10^{-06}$ & $6.09 \times 10^{-07}$ & 0.7573 & 0.191 \\
\hline
\end{tabular}




\subsubsection{Case I (Maximum Number of Iterations $=500$ )}

In this case, the general control parameters of algorithms, such as the number of candidate solutions and the maximum number of iterations were chosen the same as those given by [36]. In Table 4, simulation results for 30 candidate solutions and the maximum number of iterations $=500$ are presented. For the verification of the results, the proposed MALO algorithm was compared to the basic ALO algorithm, well-known and recent algorithms, such as the Whale Optimization Algorithm (WOA) [36], Gravitational Search Algorithm (GSA) [38], PSO [39] and Archimedes optimization algorithm (AOA) [40]. The results of WOA, GSA, and PSO were obtained from [36] while MALO and basic ALO are implemented based on the same parameters which are taken from [33] and AOA is implemented based on the parameters which are mentioned in its original paper [40].

Unimodal functions $\left(F_{1}-F_{7}\right)$ have one global minimum. These functions are sufficient for testing the convergence rate and the exploitation capability of algorithms. It can be inferred from Table 4 that the MALO algorithm is very competitive with basic ALO, WOA, GSA, PSO, and AOA. In particular, for $F_{1}-F_{4}$, only MALO can provide the exact optimum value. Moreover, the MALO algorithm shows the best optimization' performance for functions $F_{5}$ and $F_{7}$ in terms of average and standard deviation. As a result, the MALO algorithm has a high exploitation capability.

In contrast to unimodal functions, multimodal functions $\left(F_{8}-F_{13}\right)$ have a large number of local minima. As a result, these kinds of benchmark functions are better for testing the exploration capability and local optima avoidance of algorithms. Fixed-dimensional multimodal functions $\left(F_{14}-F_{23}\right)$ have a pre-defined number of design variables and provide different search spaces compared to multimodal functions.

Table 4 shows that MALO, WOA, and AOA provide the exact optimum value for multimodal function $F_{9}$ while MALO and AOA achieve the exact optimum value for $F_{11}$. For $F_{10}, F_{12} F_{13}, F_{14}, F_{21}, F_{22}$; the MALO algorithm is better than ALO, WOA, GSA, PSO, and AOA in terms of average and standard deviation.

Moreover, the MALO algorithm is competitive with GSA for function $F_{23}$ in terms of average and standard deviation. Thus, MALO has also a high exploration capability which leads this algorithm to explore the promising areas without any disruption. In this case, out of twenty-three benchmark functions, MALO achieves the best results for fourteen functions, while GSA for five, basic ALO for three, WOA for three, AOA for three, PSO for two. The convergence curves of the ALO and MALO for some of the functions by considering the maximum number of iterations $=500$ are shown in Figure 4 . The iterations are shown on the horizontal axis while the average function values are shown on the vertical axis. As can be observed, the proposed MALO algorithm escapes the local optima and provides a better convergence rate as compared to the basic ALO algorithm.

\subsubsection{Case II (Maximum Number of Iterations $=1000$ )}

In this case, the number of candidate solutions and the maximum number of iterations were selected the same as those given by [33]. Results for 30 candidate solutions and the maximum number of iterations $=1000$ are shown in Tables 5 and 6 . Nine optimization algorithms, including the basic ALO algorithm, Bat Algorithm (BA) [41], States of Matter Search (SMS) [42,43], Particle Swarm Optimization (PSO) [39], Flower Pollination Algorithm (FPA) [44], Genetic Algorithms (GA) [45], Firefly Algorithm (FA) [46,47], Cuckoo Search (CS) [48] and Archimedes optimization algorithm (AOA) [40] were investigated in order to compare the optimization results. Most of the comparative algorithms' results were taken from [33].

As can be seen from Table 5, the MALO algorithm is very competitive with the basic ALO algorithm, BA, SMS, PSO, FPA, GA, FA, CS, and AOA in optimizing the unimodal functions. MALO achieves exact optimum results for $F_{1}-F_{4}$ and is the best efficient optimizer for functions $F_{5}$ and $F_{7}$. Hence, the MALO algorithm has a high exploitation capability for the maximum number of iterations $=1000$. 

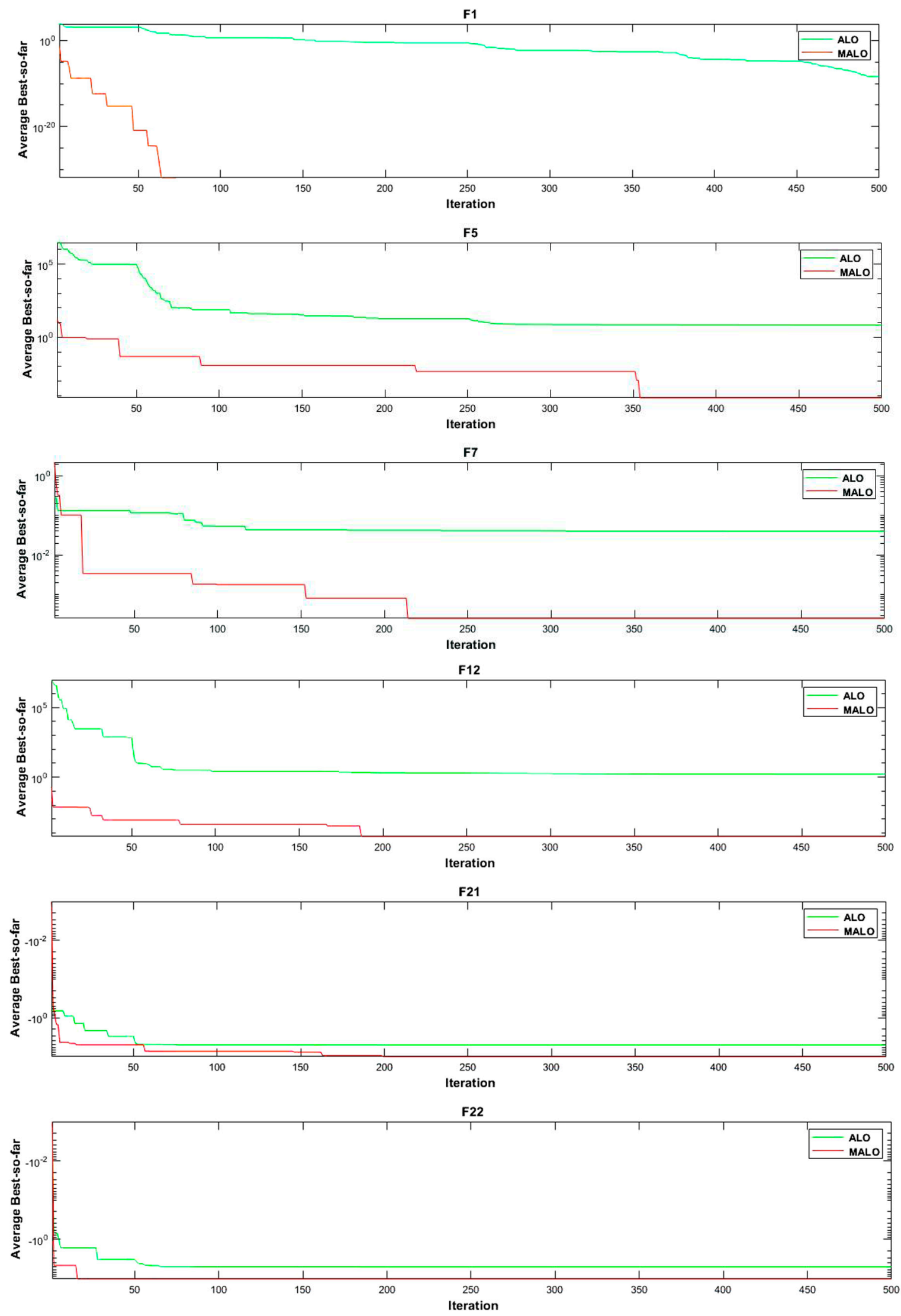

Figure 4. Convergence curves of ALO and MALO on six of the benchmark functions (500 iterations). 
Table 6 shows the results of multimodal functions. MALO algorithm is the second efficient optimizer for function $F_{8}$. Moreover, for $F_{9}$ only MALO gives the exact optimum value and for $F_{11}$, MALO and AOA achieve the exact optimum value. MALO and AOA are the most efficient optimizers for $F_{10}$, in terms of average and standard deviation. Therefore, MALO has also a high exploration capability for the maximum number of iterations $=1000$. In this case, out of thirteen benchmark functions, MALO provides optimum results for nine functions, basic ALO achieves good results for three functions, AOA shows good results for three functions, while other algorithms fail to provide better results. Figure 5 provides the convergence curves of the ALO and MALO for some of the functions by considering the maximum number of iterations $=1000$. As illustrated, the proposed MALO algorithm escapes the local optima with a high convergence rate.
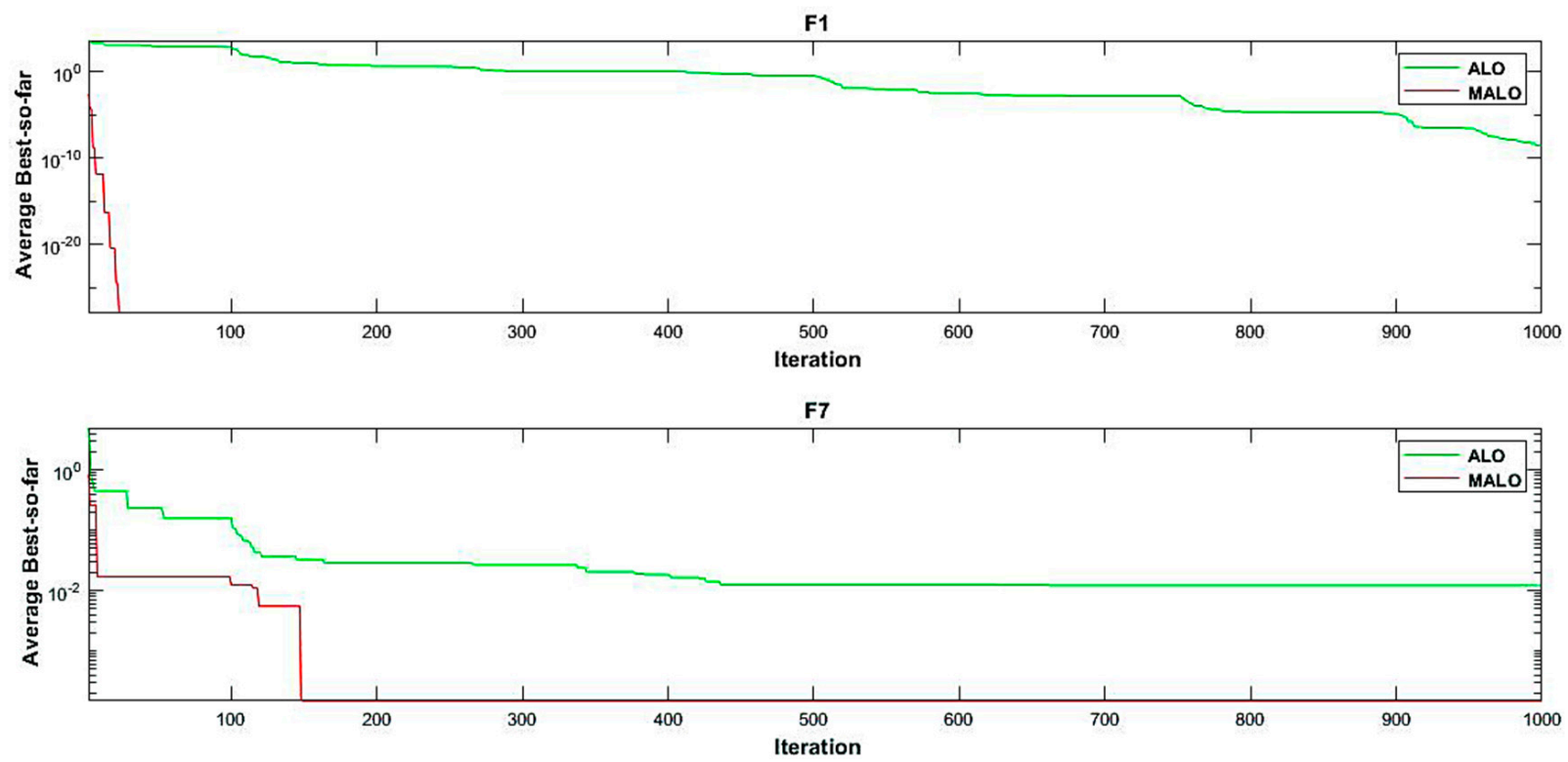

Figure 5. Convergence curves of ALO and MALO on some of the benchmark functions (1000 iterations).

\subsection{Experiment 2: Results of Malo on Balanced and Imbalanced Real-World Datasets}

In this section, some state-of-the-art methods were used in comparison with the proposed method, and the experimental results are recorded. The experiments were performed on two scenarios: The first scenario contains experiments on balanced and imbalanced real-world datasets in their original form. The second scenario contains experiments on oversampling imbalanced datasets. In these experiments, reduced datasets were classified by SVM in all experiments and the datasets with different numbers of instances and attributes were chosen from the UCI machine learning repository [49] to assess the classification performance of the MALO algorithm on balanced and imbalanced data. Tables 7 and 8 show the characteristics of the datasets.

All experiments on data were conducted by dividing the instances of each dataset randomly into three sets: training, testing, and validation sets. Hibernation between the proposed MALO algorithm and SVM classifier was applied to perform an instance reduction of the training set and 30 runs of the algorithm were performed independently to record the performance measures Accuracy, BACC, G-mean, AUC, and the run time for each test set.

\subsubsection{First Scenario}

This scenario was designed to assess the effectiveness of the proposed instance reduction method on both balanced and imbalanced datasets in their original form. The proposed method was implemented, and some experiments were conducted to enhance 
overall performance by removing redundant instances to obtain better values for four evaluation measures: Accuracy, BACC, G-mean, and AUC in addition to the run time.

Table 7. Balanced and imbalanced datasets characteristics.

\begin{tabular}{|c|c|c|c|c|}
\hline \multicolumn{2}{|c|}{ Dataset } & \multirow{2}{*}{$\begin{array}{c}\text { \#Instances } \\
286\end{array}$} & \multirow{2}{*}{$\begin{array}{c}\text { \#Features } \\
10\end{array}$} & \multirow{2}{*}{$\begin{array}{c}\begin{array}{c}\text { Imbalance } \\
\text { Ratio }\end{array} \\
2.33\end{array}$} \\
\hline 1 & Breast cancer & & & \\
\hline 2 & Parkinsons & 195 & 22 & 3.06 \\
\hline 3 & Crx & 307 & 15 & 1.20 \\
\hline 4 & vehicle1 & 946 & 18 & 3.36 \\
\hline 5 & vehicle2 & 946 & 18 & 3.19 \\
\hline 6 & Heart & 267 & 13 & 1.27 \\
\hline 7 & glass0 & 214 & 9 & 12.00 \\
\hline 8 & Ionosphere & 351 & 34 & 1.78 \\
\hline 9 & Breast_tissue & 106 & 9 & 1.94 \\
\hline 10 & Tic-tac-toe & 958 & 9 & 1.86 \\
\hline 11 & Pima & 768 & 8 & 1.86 \\
\hline 12 & Wdbc & 569 & 30 & 1.70 \\
\hline 13 & Liver & 345 & 6 & 1.38 \\
\hline 14 & Wisconsin & 699 & 9 & 1.86 \\
\hline 15 & Bupa & 345 & 6 & 1.38 \\
\hline
\end{tabular}

Table 8. Imbalanced datasets characteristics.

\begin{tabular}{|c|c|c|c|c|}
\hline \multicolumn{2}{|c|}{ Dataset } & \multirow{2}{*}{$\begin{array}{c}\text { \#Instances } \\
208\end{array}$} & \multirow{2}{*}{$\begin{array}{c}\text { \#Features } \\
60\end{array}$} & \multirow{2}{*}{$\begin{array}{c}\begin{array}{c}\text { Imbalance } \\
\text { Ratio }\end{array} \\
1.28\end{array}$} \\
\hline 1 & Sonar & & & \\
\hline 2 & Ecoli (im-others) & 336 & 7 & 3.35 \\
\hline 3 & Ionsphere & 351 & 34 & 1.78 \\
\hline 4 & Pid & 768 & 8 & 1.86 \\
\hline 5 & $\begin{array}{c}\text { Segment } \\
\text { (BRICKFACE- } \\
\text { others) }\end{array}$ & 2310 & 19 & 6.14 \\
\hline 6 & $\begin{array}{c}\text { Libra } \\
\text { (123-others) }\end{array}$ & 360 & 90 & 5 \\
\hline 7 & $\begin{array}{c}\text { Libra } \\
\text { (456-others) }\end{array}$ & 360 & 90 & 5 \\
\hline 8 & $\begin{array}{c}\text { Vehicle } \\
\text { (van-others) }\end{array}$ & 846 & 18 & 3.25 \\
\hline 9 & $\begin{array}{c}\text { Glass (tableware- } \\
\text { others) }\end{array}$ & 214 & 9 & 22.78 \\
\hline 10 & Wine (1-others) & 178 & 13 & 2.03 \\
\hline 11 & Wine (3-others) & 178 & 13 & 2.7 \\
\hline 12 & $\begin{array}{c}\text { Yeast } \\
\text { (POX-others) }\end{array}$ & 1484 & 8 & 99 \\
\hline 13 & $\begin{array}{c}\text { Yeast } \\
\text { (ME2-others) }\end{array}$ & 1484 & 8 & 32.33 \\
\hline 14 & Abalone (18-9) & 731 & 8 & 16.4 \\
\hline 15 & Vowel (1-others) & 528 & 10 & 10.11 \\
\hline 16 & Vowel (2-others) & 528 & 10 & 10.11 \\
\hline 17 & Vowel (3-others) & 528 & 10 & 10.11 \\
\hline 18 & German & 1000 & 24 & 2.33 \\
\hline
\end{tabular}

Table 9 presents the overall performance of the MALO instance reduction method, compared with the basic ALO algorithm and two state-of-the-art instance reduction techniques: Gray Wolf algorithm (GWO) and Whale Optimization (WOA). The results in Table 9 show clearly that the MALO outperforms these methods in terms of the classification accuracy 
and BACC values on almost all balanced and imbalanced datasets (12 datasets out of 15) which proves the stability of our proposal against other instance reduction methods.

Table 9. Performances of MALO, ALO, GWO, and WOA on balanced and imbalanced realworld datasets.

\begin{tabular}{|c|c|c|c|c|c|c|c|c|c|}
\hline Dataset & ALO & MALO & GWO & WOA & Dataset & ALO & MALO & GWO & WOA \\
\hline $\begin{array}{l}\text { Breast } \\
\text { cancer }\end{array}$ & & & & & Wisconsin & & & & \\
\hline Accuracy & 75.36 & 79.71 & 79.71 & 78.26 & Accuracy & 95.88 & 96.47 & 95.88 & 95.88 \\
\hline BACC & 73.62 & 76.23 & 74.78 & 75.36 & BACC & 95.65 & 96 & 95.88 & 95.53 \\
\hline G-mean & 53.07 & 69.47 & 65.00 & 63.57 & G-mean & 96.05 & 96.5 & 96.05 & 96.05 \\
\hline AUC & 61.94 & 71.38 & 69.44 & 67.40 & AUC & 96.05 & 96.5 & 96.05 & 96.05 \\
\hline $\begin{array}{l}\text { Time } \\
\text { Crx }\end{array}$ & 0.0232 & 0.0202 & 0.0165 & 0.0231 & $\begin{array}{l}\text { Time } \\
\text { Bupa }\end{array}$ & 0.0351 & 0.0236 & 0.0188 & 0.0264 \\
\hline Accuracy & 65.64 & 66.26 & 65.64 & 64.42 & Accuracy & 63.49 & 61.86 & 65.12 & 63.26 \\
\hline $\mathrm{BACC}$ & 63.31 & 63.44 & 62.82 & 61.96 & BACC & 67.44 & 68.6 & 67.44 & 69.77 \\
\hline G-mean & 64.64 & 65.39 & 64.10 & 64.45 & G-mean & 57.83 & 60.83 & 59.16 & 59.81 \\
\hline AUC & 65.01 & 65.68 & 64.78 & 64.45 & AUC & 63.06 & 64.06 & 63.06 & 65.44 \\
\hline Time & 0.0277 & 0.0248 & 0.0328 & 0.0296 & Time & 0.0343 & 0.0238 & 0.0193 & 0.0276 \\
\hline Heart & & & & & Breast_tissue & & & & \\
\hline Accuracy & 70.15 & 71.64 & 71.64 & 67.16 & Accuracy & 75.38 & 78.46 & 69.23 & 70 \\
\hline BACC & 65.37 & 65.67 & 64.78 & 65.67 & BACC & 80.77 & 84.62 & 80.77 & 73.08 \\
\hline G-mean & 68.58 & 69.75 & 70.46 & 67.12 & G-mean & 76.7 & 82.84 & 72.31 & 62.62 \\
\hline AUC & 69.19 & 70.54 & 70.86 & 67.12 & AUC & 77.45 & 83.01 & 74.84 & 66.34 \\
\hline Time & 0.0339 & 0.0240 & 0.0258 & 0.0253 & Time & 0.0260 & 0.0228 & 0.0253 & 0.0282 \\
\hline Ionosphere & & & & & glass0 & & & & \\
\hline Accuracy & 95.40 & 96.55 & 96.55 & 95.40 & Accuracy & 77.74 & 80 & 78.49 & 79.62 \\
\hline BACC & 94.25 & 94.94 & 94.48 & 94.71 & BACC & 81.13 & 83.02 & 81.13 & 81.13 \\
\hline G-mean & 93.33 & 95.04 & 95.04 & 93.33 & G-mean & 79.83 & 81.15 & 80.03 & 79.83 \\
\hline AUC & 93.55 & 95.16 & 95.16 & 93.55 & AUC & 79.9 & 81.29 & 80.07 & 79.9 \\
\hline Time & 0.0272 & 0.0242 & 0.0271 & 0.0271 & Time & 0.0272 & 0.0233 & 0.0259 & 0.0251 \\
\hline Tic-tac-toe & & & & & vehicle1 & & & & \\
\hline Accuracy & 99.16 & 98.47 & 98.47 & 98.47 & Accuracy & 75.64 & 76.4 & 76.11 & 75.26 \\
\hline BACC & 98.58 & 98.74 & 98.66 & 98.66 & BACC & 76.78 & 77.25 & 76.78 & 76.3 \\
\hline G-mean & 98.79 & 98.18 & 98.18 & 98.18 & G-mean & 53.55 & 55.48 & 57.88 & 50.48 \\
\hline AUC & 98.80 & 98.19 & 98.19 & 98.19 & AUC & 61.28 & 62.85 & 63.74 & 59.78 \\
\hline $\begin{array}{l}\text { Time } \\
W d b c\end{array}$ & 0.0520 & 0.0274 & 0.0319 & 0.0373 & $\begin{array}{c}\text { Time } \\
\text { vehicle2 }\end{array}$ & 0.0333 & 0.0261 & 0.0276 & 0.0335 \\
\hline Accuracy & 96.48 & 97.81 & 96.48 & 96.48 & Accuracy & 93.18 & 93.46 & 92.99 & 93.18 \\
\hline BACC & 94.51 & 95.63 & 95.21 & 94.93 & BACC & 93.84 & 93.84 & 93.36 & 94.31 \\
\hline G-mean & 96.43 & 96.99 & 96.43 & 96.43 & G-mean & 89.82 & 90.12 & 89.82 & 90.41 \\
\hline AUC & 96.43 & 96.99 & 96.43 & 96.43 & AUC & 90.07 & 90.39 & 90.07 & 90.71 \\
\hline Time & 0.0429 & 0.0250 & 0.0277 & 0.0288 & Time & 0.0353 & 0.0323 & 0.0356 & 0.0349 \\
\hline Pima & & & & & Parkinsons & & & & \\
\hline Accuracy & 69.27 & 69.79 & 69.27 & 69.79 & Accuracy & 85.42 & 87.5 & 88.33 & 85 \\
\hline $\mathrm{BACC}$ & 67.81 & 68.96 & 68.02 & 68.54 & $\mathrm{BACC}$ & 87.5 & 89.58 & 91.67 & 87.5 \\
\hline G-mean & 63.41 & 57.63 & 58.18 & 63.08 & G-mean & 79.35 & 82.92 & 81.65 & 75.31 \\
\hline AUC & 64.01 & 62.26 & 62.15 & 65.03 & AUC & 80.56 & 83.33 & 83.33 & 77.78 \\
\hline Time & 0.0314 & 0.0327 & 0.0357 & 0.0328 & Time & 0.0515 & 0.0232 & 0.0199 & 0.0258 \\
\hline Liver & & & & & & & & & \\
\hline Accuracy & 72.09 & 74.42 & 69.77 & \multicolumn{6}{|c|}{73.62} \\
\hline $\mathrm{BACC}$ & 69.77 & 71.40 & 67.44 & \multicolumn{6}{|c|}{68.84} \\
\hline G-mean & 70.24 & 69.92 & 63.90 & \multicolumn{6}{|c|}{66.67} \\
\hline AUC & 70.33 & 71.78 & 65.83 & \multicolumn{6}{|c|}{69.61} \\
\hline Time & 0.0272 & 0.0239 & 0.0259 & \multicolumn{6}{|c|}{0.0270} \\
\hline
\end{tabular}

It can also be noticed from Table 9 that our proposed instance reduction method ranks top in G-mean and AUC (9 datasets out of 15), where it provides a significantly higher number of best G-mean and AUC values than other compared methods. This superiority proves that our method has improved the trade-offs between sensitivity and specificity, which indicates the reduction obtained by our instance reduction method for false +ves and -ves, over state-of-the-art methods.

MALO algorithm also increments up to $4 \%$ in Accuracy rate, $3 \%$ in BACC rate, $15 \%$ in G-mean rate, and 9\% in AUC for "Breast cancer" dataset, over the basic ALO algorithm. 
Results in Table 9 also indicate that the proposed MALO is less than the ALO algorithm for all the datasets and has superiority for saving computational time (better in 9 datasets out of 15) while maintaining the best performance over the other compared algorithms. The convergence curves between the fitness function and iterations for the ALO, MALO, GWO, and WOA for the glass 0 and Breast_tissue datasets are shown in Figure 6. The iterations are shown on the horizontal axis while the fitness function values are shown on the vertical axis. As can be observed, the proposed MALO algorithm provides a better convergence rate as compared to the basic ALO algorithm and other compared algorithms.
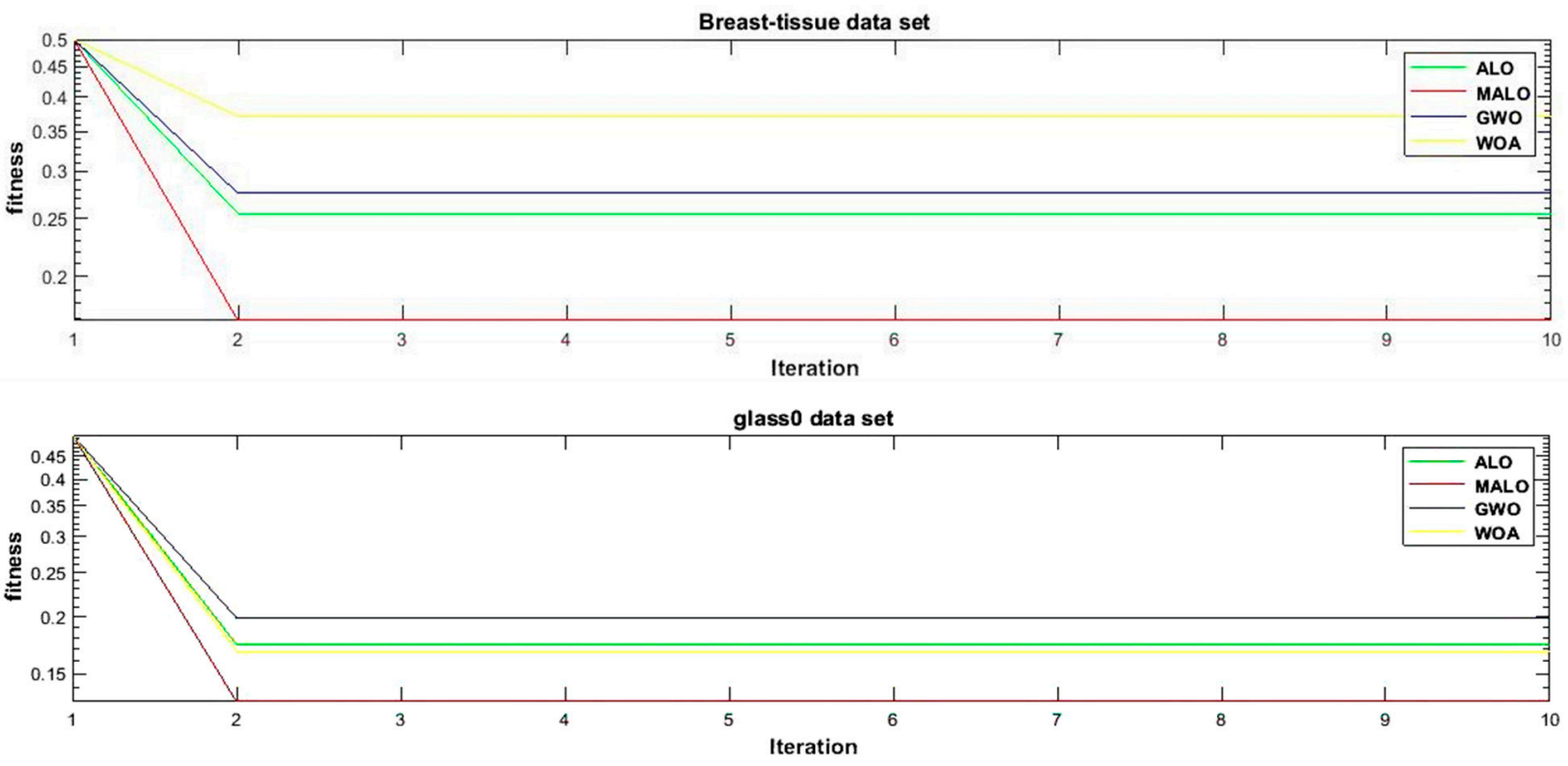

Figure 6. Convergence curves of ALO, MALO, GWO, and WOA on two datasets.

To perform the comparison, the non-parametric statistical hypothesis Wilcoxon signedrank test (a paired difference, two-sided signed-rank test) [50] was used to perform a statistical significance analysis and derive fairly strong conclusions. All the methods were compared with MALO for each dataset. For each two compared methods, the differences were calculated and ranked from 1 (smallest) to 15 (largest). The signs (' + ' or '-') were subsequently assigned to the corresponding differences of the ranks. $\mathrm{R}+$ and $\mathrm{R}-$ were assigned to all the +ve and - ve ranks after summing up separately, respectively. The $\mathrm{T}$ value was compared against a significance level $\alpha=0.05$, with a critical value, equals 25 for 15 datasets where $\mathrm{T}=\min \{\mathrm{R}+, \mathrm{R}-\}$. The null hypothesis was that all performance differences between any two compared methods may occur by chance and the null hypothesis was rejected only if the $\mathrm{T}$ value is $<$ or $=$ to 25 (the critical value).

Tables 10-17 present the significance test results and the addition symbol ' + ' in Tables 14-17 indicate that our proposal MALO outperforms the compared methods.

The G-mean comparison: Table 10 shows the significance test results of average G-mean for MALO vs. ALO, MALO vs. GWO, and MALO vs. WOA, using the SVM classifier. In the case of MALO vs. ALO, MALO is better (+ve difference) than ALO for 12 datasets, while ALO is better (-ve difference) than MALO for three datasets. After calculating the total of + ve ranks $R_{+}=100$ and the total - ve ranks $R-=20$. As 15 datasets were used, the $\mathrm{T}$ value at the significance level of 0.05 should be $\leq 25$ to reject a null hypothesis. We can conclude that MALO can statistically outperform ALO as $\mathrm{T}=\min \{\mathrm{R}+, \mathrm{R}-\}=\min \{100,20\}=20<25$. Likewise, in the case of MALO vs. GWO, MALO can statistically outperform the GWO as $\mathrm{T}=\min \{95,24\}=24<25$.

In the case of MALO vs. WOA, MALO obtains better differences for 13 datasets, while WOA just obtains better differences for two datasets. We can conclude that MALO can statistically outperform WOA as $\mathrm{T}=\min \{106,14\}=14<25$. 
Table 10. Significance test of average g-mean between MALO vs. ALO, MALO vs. GWO, and MALO vs. WOA.

\begin{tabular}{|c|c|c|c|c|c|c|c|c|c|c|c|c|}
\hline Dataset & MALO & ALO & Difference & Rank & MALO & GWO & Difference & Rank & MALO & WOA & Difference & Rank \\
\hline $\begin{array}{l}\text { Breast } \\
\text { cancer }\end{array}$ & 69.47 & 53.07 & +16.4 & 15 & 69.47 & 65 & +4.47 & 13 & 69.47 & 63.57 & +5.9 & 13 \\
\hline Crx & 65.39 & 64.64 & +0.75 & 6 & 65.39 & 64.1 & +1.29 & 9 & 65.39 & 64.45 & +0.94 & 5 \\
\hline Heart & 69.75 & 68.58 & +1.17 & 7 & 69.75 & 70.46 & -0.71 & -7 & 69.75 & 67.12 & +2.63 & 9 \\
\hline Ionosphere & 95.04 & 93.33 & +1.71 & 9 & 95.04 & 95.04 & 0 & 1 & 95.04 & 93.33 & +1.71 & 8 \\
\hline $\begin{array}{l}\text { Tic-tac- } \\
\text { toe }\end{array}$ & 98.18 & 98.79 & -0.61 & -5 & 98.18 & 98.18 & 0 & 1 & 98.18 & 98.18 & 0 & 1 \\
\hline Wdbc & 96.99 & 96.43 & +0.56 & 4 & 96.99 & 96.43 & +0.56 & 6 & 96.99 & 96.43 & +0.56 & 4 \\
\hline Pima & 57.63 & 63.41 & -5.78 & -13 & 57.63 & 58.18 & -0.55 & -5 & 57.63 & 63.08 & -5.45 & -12 \\
\hline Liver & 69.92 & 70.24 & -0.32 & -2 & 69.92 & 63.9 & +6.02 & 14 & 69.92 & 66.67 & +3.25 & 10 \\
\hline Wisconsin & 96.5 & 96.05 & +0.45 & 3 & 96.5 & 96.05 & +0.45 & 4 & 96.5 & 96.05 & +0.45 & 3 \\
\hline Bupa & 60.83 & 57.83 & +3 & 12 & 60.83 & 59.16 & +1.67 & 10 & 60.83 & 59.81 & +1.02 & 6 \\
\hline Breast_tissue & e 82.84 & 76.7 & +6.14 & 14 & 82.84 & 72.31 & +10.53 & 15 & 82.84 & 62.62 & +20.22 & 15 \\
\hline glass 0 & 81.15 & 79.83 & +1.32 & 8 & 81.15 & 80.03 & +1.12 & 8 & 81.15 & 79.83 & +1.32 & 7 \\
\hline vehicle1 & 55.48 & 53.55 & +1.93 & 10 & 55.48 & 57.88 & -2.4 & -12 & 55.48 & 50.48 & +5 & 11 \\
\hline vehicle2 & 90.12 & 89.82 & +0.3 & 1 & 90.12 & 89.82 & +0.3 & 3 & 90.12 & 90.41 & -0.29 & -2 \\
\hline Parkinsons & 83.33 & 80.56 & +2.77 & 11 & 83.33 & 81.65 & +1.68 & 11 & 83.33 & 75.31 & +8.02 & 14 \\
\hline \multicolumn{6}{|c|}{$\mathrm{T}=\min \{100,20\}=20$} & \multicolumn{4}{|c|}{$\mathrm{T}=\min \{95,24\}=24$} & \multicolumn{3}{|c|}{$\mathrm{T}=\min \{106,14\}=14$} \\
\hline
\end{tabular}

Table 11. Significance test of average accuracy between MALO vs. ALO, MALO vs. GWO, and MALO vs. WOA.

\begin{tabular}{|c|c|c|c|c|c|c|c|c|c|c|c|c|}
\hline Dataset & MALO & ALO & Difference & Rank & MALO & GWO & Difference & Rank & MALO & WOA & Difference & Rank \\
\hline $\begin{array}{l}\text { Breast } \\
\text { cancer }\end{array}$ & 79.71 & 75.36 & +4.35 & 15 & 79.71 & 79.71 & 0 & 1 & 79.71 & 78.26 & +1.45 & 11 \\
\hline Crx & 66.26 & 65.64 & +0.62 & 4 & 66.26 & 65.64 & +0.62 & 9 & 66.26 & 64.42 & +1.84 & 12 \\
\hline Heart & 71.64 & 70.15 & +1.49 & 9 & 71.64 & 71.64 & 0 & 1 & 71.64 & 67.16 & +4.48 & 14 \\
\hline Ionosphere & 96.55 & 95.4 & +1.15 & 7 & 96.55 & 96.55 & 0 & 1 & 96.55 & 95.4 & +1.15 & 8 \\
\hline $\begin{array}{l}\text { Tic-tac- } \\
\text { toe }\end{array}$ & 98.47 & 99.16 & -0.69 & -5 & 98.47 & 98.47 & 0 & 1 & 98.47 & 98.47 & 0 & 1 \\
\hline Wdbc & 97.81 & 96.48 & +1.33 & 8 & 97.81 & 96.48 & +1.33 & 11 & 97.81 & 96.48 & +1.33 & 9 \\
\hline Pima & 69.79 & 69.27 & +0.52 & 2 & 69.79 & 69.27 & +0.52 & 7 & 69.79 & 69.79 & 0 & 1 \\
\hline Liver & 74.42 & 72.09 & +2.33 & 13 & 74.42 & 69.77 & +4.65 & 14 & 74.42 & 73.62 & +0.8 & 6 \\
\hline Wisconsin & 96.47 & 95.88 & +0.59 & 3 & 96.47 & 95.88 & +0.59 & 8 & 96.47 & 95.88 & +0.59 & 5 \\
\hline Bupa & 61.86 & 63.49 & -1.63 & -10 & 61.86 & 65.12 & -3.26 & -13 & 61.86 & 63.26 & -1.4 & -10 \\
\hline Breast_tissu & le78.46 & 75.38 & +3.08 & 14 & 78.46 & 69.23 & +9.23 & 15 & 78.46 & 70 & +8.46 & 15 \\
\hline glass 0 & 80 & 77.74 & +2.26 & 12 & 80 & 78.49 & +1.51 & 12 & 80 & 79.62 & +0.38 & 4 \\
\hline vehicle1 & 76.4 & 75.64 & +0.76 & 6 & 76.4 & 76.11 & +0.29 & 5 & 76.4 & 75.26 & +1.14 & 7 \\
\hline vehicle2 & 93.46 & 93.18 & +0.28 & 1 & 93.46 & 92.99 & +0.47 & 6 & 93.46 & 93.18 & +0.28 & 3 \\
\hline Parkinsons & 87.5 & 85.42 & +2.08 & 11 & 87.5 & 88.33 & -0.83 & -10 & 87.5 & 85 & +2.5 & 13 \\
\hline \multicolumn{6}{|c|}{$\mathrm{T}=\min \{105,15\}=15$} & \multicolumn{4}{|c|}{$\mathrm{T}=\min \{91,23\}=23$} & \multicolumn{3}{|c|}{$\mathrm{T}=\min \{109,10\}=10$} \\
\hline
\end{tabular}

The $\mathrm{T}$ values of Table 10 are listed as summary results in Table 14, which shows that our proposed instance reduction method can statistically outperform the compared.

This result is consistent with our expectations, regarding that G-mean was considered as the fitness function in our proposed instance reduction method in these experiments.

The accuracy comparison: Table 11 shows the significance test results of average accuracy for MALO vs. ALO, MALO vs. GWO, and MALO vs. WOA, using the SVM classifier. In the case of MALO vs. ALO, MALO is better (+ve difference) than ALO for 13 datasets, while ALO is better (-ve difference) than MALO for two datasets. After calculating the total of + ve ranks $\mathrm{R}+=105$ and the total - ve ranks $\mathrm{R}-=15$. We can conclude that MALO can statistically outperform ALO as T $=\min \{105,15\}=15<25$. Likewise, in the case of MALO vs. GWO, MALO can statistically outperform the GWO as $\mathrm{T}=\min \{91,23\}=23<25$.

In the case of MALO vs. WOA, MALO obtains better differences for 14 datasets, while WOA obtains better differences for just one dataset. We can conclude that MALO can statistically outperform WOA as $\mathrm{T}=\min \{109,10\}=10<25$. 
Table 12. Significance test of average bacc between MALO vs. ALO, MALO vs. GWO, and MALO vs. WOA.

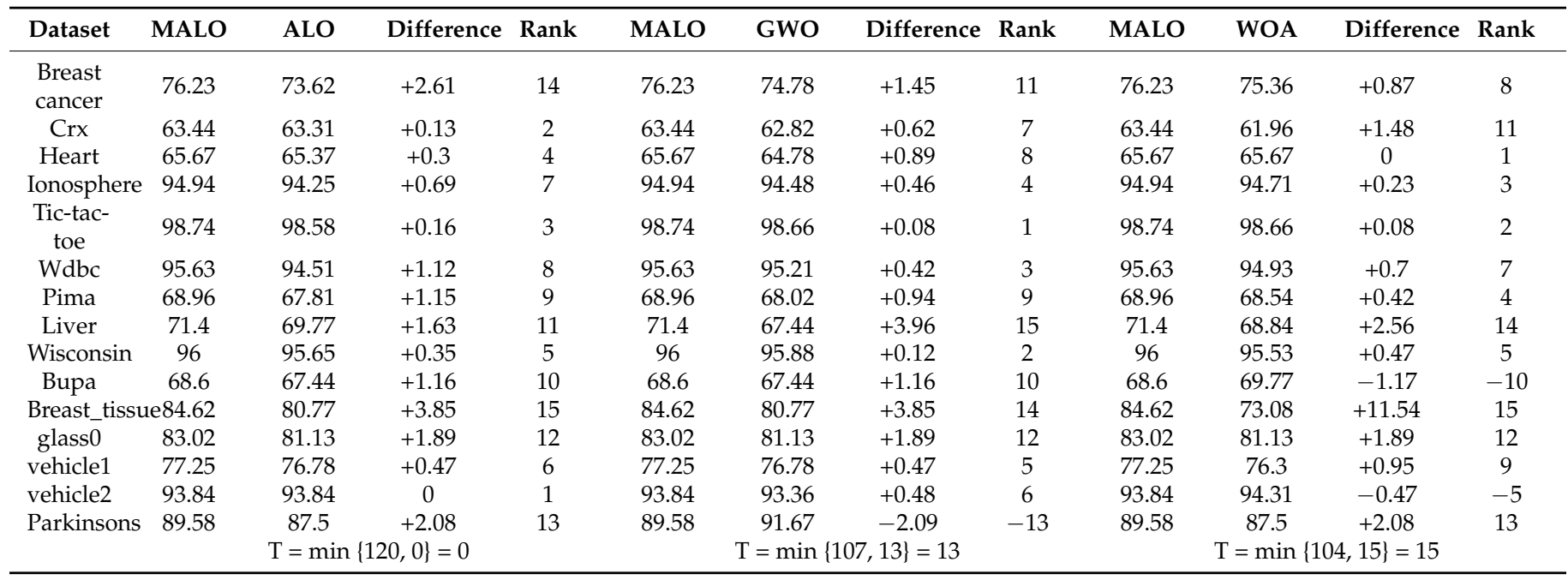

Table 13. Significance test of average auc between MALO vs. ALO, MALO vs. GWO, and MALO vs. WOA.

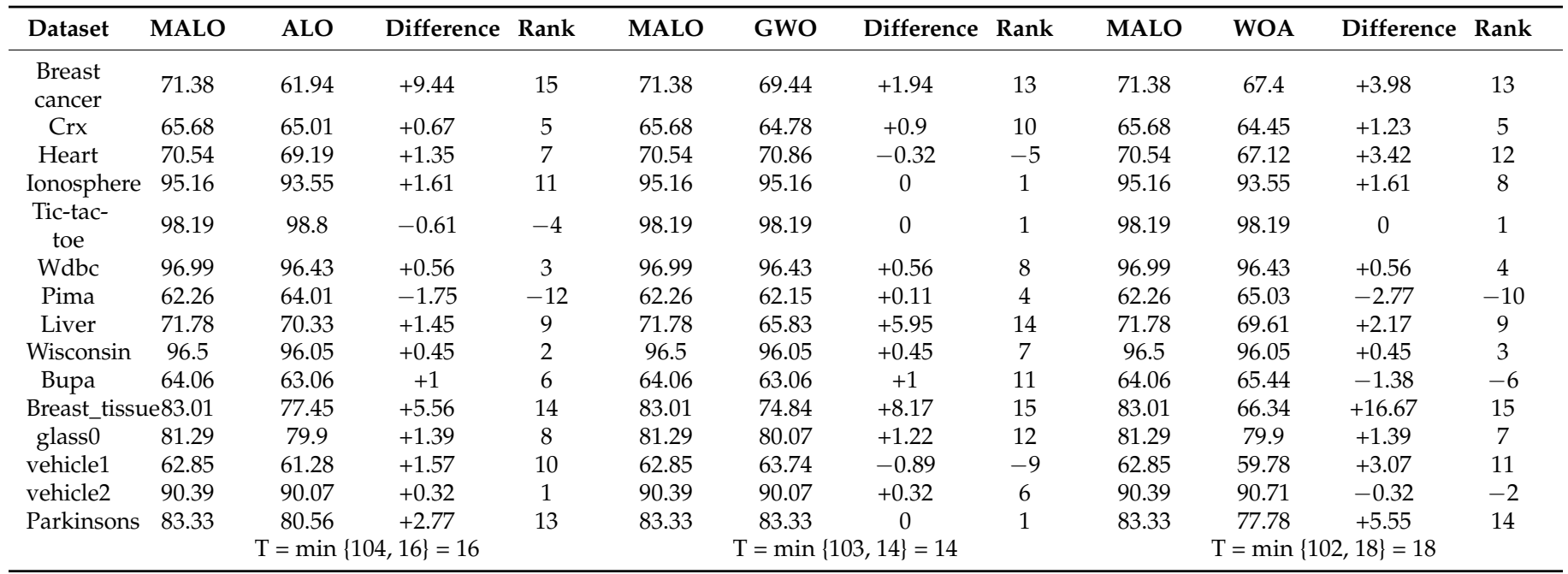

Table 14. The $t$ values of significance test on averaged g-mean between MALO vs. ALO, MALO vs. GWO, and MALO vs. WOA.

\begin{tabular}{cccc}
\hline & MALO vs. ALO & MALO vs. GWO & MALO vs. WOA \\
\hline $\mathrm{R}+$ & 100 & 95 & 106 \\
$\mathrm{R}-$ & 20 & 24 & 14 \\
$\mathrm{~T}=\min \{\mathrm{R}+, \mathrm{R}-\}$ & $\mathbf{2 0}(+)$ & $\mathbf{2 4}(+)$ & $\mathbf{1 4}(+)$ \\
\hline
\end{tabular}

Table 15. The $t$ values of significance test on averaged accuracy between MALO VS. ALO, MALO vs. GWO, and MALO vs. WOA.

\begin{tabular}{cccc}
\hline & MALO vs. ALO & MALO vs. GWO & MALO vs. WOA \\
\hline $\mathrm{R}+$ & 105 & 91 & 109 \\
$\mathrm{R}-$ & 15 & 23 & 10 \\
$\mathrm{~T}=\min \{\mathrm{R}+, \mathrm{R}-\}$ & $\mathbf{1 5}(+)$ & $\mathbf{2 3}(+)$ & $\mathbf{1 0}(+)$ \\
\hline
\end{tabular}


Table 16. The $t$ values of significance test on averaged bacc between MALO vs. ALO, MALO vs. GWO, and MALO vs. WOA.

\begin{tabular}{cccc}
\hline & MALO vs. ALO & MALO vs. GWO & MALO vs. WOA \\
\hline $\mathrm{R}+$ & 120 & 107 & 104 \\
$\mathrm{R}-$ & 0 & 13 & 15 \\
$\mathrm{~T}=\min \{\mathrm{R}+, \mathrm{R}-\}$ & $\mathbf{0}(+)$ & $\mathbf{1 3}(+)$ & $\mathbf{1 5}(+)$ \\
\hline
\end{tabular}

Table 17. The $t$ values of significance test on averaged auc between MALO vs. ALO, MALO vs. GWO, and MALO vs. WOA.

\begin{tabular}{cccc}
\hline & MALO vs. ALO & MALO vs. GWO & MALO vs. WOA \\
\hline $\mathrm{R}+$ & 104 & 103 & 102 \\
$\mathrm{R}-$ & 16 & 14 & 18 \\
$\mathrm{~T}=\min \{\mathrm{R}+, \mathrm{R}-\}$ & $\mathbf{1 6}(+)$ & $\mathbf{1 4}(+)$ & $\mathbf{1 8}(+)$ \\
\hline
\end{tabular}

The $\mathrm{T}$ values of Table 11 are listed as summary results in Table 15, which shows that our proposed instance reduction method can statistically outperform the compared methods according to the average accuracy values.

The BACC comparison: Table 12 shows the significance test results of average BACC for MALO vs. ALO, MALO vs. GWO, and MALO vs. WOA, using the SVM classifier. In the case of MALO vs. ALO, MALO is better (+ve difference) than ALO for all datasets. After calculating the total of + ve ranks $\mathrm{R}+=120$ and the total - ve ranks $\mathrm{R}-=0$. We can conclude that MALO can statistically outperform ALO as $\mathrm{T}=\min \{120,0\}=0<25$. In the case of MALO vs. GWO, MALO obtains better differences for 14 datasets, while GWO obtains better differences for just one dataset. We can conclude that MALO can statistically outperform GWO as $\mathrm{T}=\min \{107,13\}=13<25$. In the case of MALO vs. WOA, MALO obtains better differences for 13 datasets, while WOA just obtains better differences for two datasets. We can conclude that MALO can statistically outperform WOA as $\mathrm{T}=\min \{104,15\}=15<25$.

The $\mathrm{T}$ values of Table 12 are listed as summary results in Table 16, which shows that our proposed instance reduction method can statistically outperform the compared methods according to the average BACC values.

The AUC comparison: Table 13 shows the significance test results of average AUC for MALO vs. ALO, MALO vs. GWO, and MALO vs. WOA, using the SVM classifier. In the case of MALO vs. ALO, MALO is better (+ve difference) than ALO for 13 datasets, while ALO is better (-ve difference) than MALO for two datasets. After calculating the total of + ve ranks $\mathrm{R}+=104$ and the total - ve ranks $\mathrm{R}-=16$. We can conclude that MALO can statistically outperform ALO as T $=\min \{104,16\}=16<25$. Likewise, in the case of MALO vs. GWO, MALO can statistically outperform the GWO as $\mathrm{T}=\min \{103,14\}=14<25$. In the case of MALO vs. WOA, MALO obtains better differences for 12 datasets, while WOA is better than MALO for three datasets. We can conclude that MALO can statistically outperform WOA as $\mathrm{T}=\min \{102,18\}=18<25$.

The $\mathrm{T}$ values of Table 13 are listed as summary results in Table 17, which shows that our proposed instance reduction method can statistically outperform the compared methods according to the average AUC values.

From the preceding results of the statistical Wilcoxon signed-rank test, we can conclude that the instance reduction proposed method can statistically outperform the ALO algorithm and has strong competitiveness against other comparative methods in terms of all used performance measures.

\subsubsection{Second Scenario}

In this scenario, the overall performance of our algorithm MALO was compared with the results obtained in [51], ALO, GWO, and WOA and tested again using $18 \mathrm{im}$ balanced datasets: as shown in Table 18 to examine the instance reduction capability of 
our proposal MALO and evaluate the effect of preprocessing the datasets using SMOTE oversampling method [34] with the MALO algorithm. Authors in [51] proposed a hybrid algorithm named ACOR or Ant Colony optimization resampling method to improve the classification performance of imbalanced datasets based on improving the existing oversampling methods' performance following two main steps: first, rebalancing the imbalanced datasets by one of four commonly used oversampling techniques (SMOTE [34], BSO [52], ADASYN [53], and ROS [54]) were used in the research; second, selecting an optimal subset from the rebalanced training datasets using ant colony optimization [55].

ACOR was experimented with using 18 real imbalanced datasets and three different classifiers the naive Bayes classifier [56], C4.5 classifier [57], and SVM-RBF (Support Vector Machine with Radial Basis Function Kernel) classifier [37,58].

In this experiment, we compared MALO with the SMOTE and ACOR-SMOTE, results obtained in [51], ALO, GWO, and WOA using the SVM-RBF classifier. The imbalanced training datasets are resampled using the common SMOTE oversampling method. Then, the proposed MALO was used to reduce the training set instances. Table 18 shows that MALO outperforms the compared methods in classification accuracy (12 imbalanced datasets out of 18), proving our technique's stability against other instance reduction methods. Additionally, results in Table 18 indicate that our proposed instance reduction method ranks top in BACC and G-mean (9 imbalanced datasets out of 18). It provides a significantly higher number of best BACC and G-mean values than other compared methods. In comparison, ACOR-SMOTE delivers better results in terms of AUC (11 imbalanced datasets out of 18). MALO algorithm also shows increment up to $7 \%$ in Accuracy rate, 3\% in BACC rate for "Sonar" dataset, 4\% in G-mean rate for "Sonar", "Wine(1-others)", and "Vowel(3-others)" datasets, and 4\% in AUC for "Sonar" dataset and "Vowel (3- others)" dataset over the basic ALO algorithm.

Figure 7 shows the box and whiskers plots for Accuracy, BACC, G-mean, and AUC of compared methods, respectively. The box and whiskers plot is a standardized way of displaying the distribution of data based on minimum value, lower quartile (25th percentile), median (50th percentile), upper quartile (75th percentile), and maximum value. The ends of the box are the lower and upper quartiles which represent the interquartile range (IQR), the two lines outside the box that extend to the minimum and maximum values are the whiskers, while the median and mean values are a horizontal line inside the box and cross sign, respectively. The box and whiskers plots in Figure 7 demonstrate that MALO outperforms the ALO in terms of all used measures except in BACC since they have almost the same performance in this term. MALO also shows a better performance in terms of accuracy and BACC than SMOTE and ACOR-SMOTE. In addition, MALO outperforms the GWO and WOA in terms of all used measures. The MALO's mean, median, and upper quartile values are very close to their perfect thresholds, indicating the proposed algorithm's stability against the compared algorithms.

Results in scenarios 1 and 2 demonstrate that MALO shows a high superiority in minimizing the amount of training set instances number, consequently maximizing the overall performance of classification compared to state-of-the-art methods used to reduce the original balanced and imbalanced datasets. Using MALO to reduce the instances of oversampled imbalanced datasets presents a good performance compared to the full oversampled dataset, the recent ACOR-SMOTE instance reduction method, ALO, GWO, and WOA.

The superiority of the instance reduction MALO algorithm in minimizing the number of imbalanced dataset instances in their original form gives it an important advantage since it improves the performance of imbalanced data without the need to perform oversampling pre-processing methods which consumes computational time and memory space. 
Table 18. Performances of smote and results in [51] compared to performances of ALO-SMOTE, MALO-SMOTE, GWO-SMOTE, and WOA-SMOTE.

\begin{tabular}{|c|c|c|c|c|c|c|c|c|c|c|c|c|c|}
\hline Dataset & SMOTE & $\begin{array}{l}\text { ACOR- } \\
\text { SMOTE }\end{array}$ & $\begin{array}{c}\text { ALO- } \\
\text { SMOTE }\end{array}$ & $\begin{array}{l}\text { MALO- } \\
\text { SMOTE }\end{array}$ & $\begin{array}{c}\text { GWO- } \\
\text { SMOTE }\end{array}$ & $\begin{array}{l}\text { WOA- } \\
\text { SMOTE }\end{array}$ & Dataset & SMOTE & $\begin{array}{l}\text { ACOR- } \\
\text { SMOTE }\end{array}$ & $\begin{array}{c}\text { ALO- } \\
\text { SMOTE }\end{array}$ & $\begin{array}{l}\text { MALO- } \\
\text { SMOTE }\end{array}$ & $\begin{array}{c}\text { GWO- } \\
\text { SMOTE }\end{array}$ & $\begin{array}{l}\text { WOA- } \\
\text { SMOTE }\end{array}$ \\
\hline Sonar & & & & & & & $\begin{array}{c}\text { Wine } \\
\text { (1-others) }\end{array}$ & & & & & & \\
\hline Accuracy & 0.72115 & 0.73558 & 0.8077 & 0.8846 & 0.8077 & 0.7885 & Accuracy & 0.97753 & 0.99438 & 0.8182 & 0.8409 & 0.8182 & 0.8409 \\
\hline BACC & 0.72184 & 0.7438 & 0.7885 & 0.8269 & 0.8015 & 0.7800 & BACC & 0.97892 & 0.98732 & 0.8318 & 0.8318 & 0.7333 & 0.7667 \\
\hline G-mean & 0.72176 & 0.7337 & 0.7519 & 0.8042 & 0.7850 & 0.7483 & G-mean & 0.97891 & 0.98731 & 0.6831 & 0.7303 & 0.6831 & 0.7303 \\
\hline $\begin{array}{c}\text { AUC } \\
\text { Ecoli } \\
\text { (im-others) }\end{array}$ & 0.82293 & 0.84768 & 0.7806 & 0.8209 & 0.8015 & 0.7800 & $\begin{array}{c}\text { AUC } \\
\text { Wine } \\
\text { (3-others) }\end{array}$ & 0.99772 & 0.99872 & 0.7333 & 0.7667 & 0.7333 & 0.7667 \\
\hline Accuracy & 0.86012 & 0.86905 & 0.8929 & 0.9048 & 0.881 & 0.8929 & Accuracy & 0.98315 & 0.99438 & 0.7045 & 0.7273 & 0.75 & 0.7045 \\
\hline BACC & 0.86364 & 0.85118 & 0.8857 & 0.8881 & 0.7844 & 0.7922 & BACC & 0.98846 & 0.99615 & 0.7273 & 0.7318 & 0.5938 & 0.4844 \\
\hline G-mean & 0.86361 & 0.85054 & 0.7698 & 0.7757 & 0.7624 & 0.7685 & G-mean & 0.98839 & 0.99615 & 0.2795 & 0.2841 & 0.4841 & 0 \\
\hline AUC & 0.93925 & 0.95262 & 0.7909 & 0.7959 & 0.7844 & 0.7922 & AUC & 0.99968 & 0.99952 & 0.5104 & 0.526 & 0.5938 & 0.4844 \\
\hline Ionsphere & & & & & & & $\begin{array}{l}\text { Yeast (POX- } \\
\text { others) }\end{array}$ & & & & & & \\
\hline Accuracy & 0.87464 & 0.91168 & 0.9425 & 0.954 & 0.9195 & 0.931 & Accuracy & 0.99124 & 0.99124 & 0.9946 & 0.9946 & 0.9919 & 0.9946 \\
\hline BACC & 0.86206 & 0.89444 & 0.931 & 0.9379 & 0.8871 & 0.9032 & BACC & 0.77363 & 0.77363 & 0.9941 & 0.9941 & 0.7 & 0.8 \\
\hline G-mean & 0.86091 & 0.89235 & 0.9158 & 0.9333 & 0.8799 & 0.898 & G-mean & 0.74061 & 0.74061 & 0.7746 & 0.7746 & 0.6325 & 0.7746 \\
\hline AUC & 0.9222 & 0.95072 & 0.9194 & 0.9355 & 0.8871 & 0.9032 & AUC & 0.69962 & 0.69962 & 0.8 & 0.8 & 0.7 & 0.8 \\
\hline Pid & & & & & & & $\begin{array}{l}\text { Yeast (ME2- } \\
\text { others }\end{array}$ & & & & & & \\
\hline Accuracy & 0.74479 & 0.76042 & 0.7031 & 0.7031 & 0.6823 & 0.6979 & Accuracy & 0.86725 & 0.8504 & 0.9865 & 0.9811 & 0.9757 & 0.9757 \\
\hline BACC & 0.73388 & 0.74415 & 0.6906 & 0.6875 & 0.5967 & 0.6087 & BACC & 0.8178 & 0.80908 & 0.9801 & 0.9784 & 0.635 & 0.5909 \\
\hline G-mean & 0.73299 & 0.7422 & 0.573 & 0.573 & 0.5252 & 0.5323 & G-mean & 0.81608 & 0.80786 & 0.7385 & 0.603 & 0.5215 & 0.4264 \\
\hline AUC & 0.82914 & 0.83262 & 0.6266 & 0.6266 & 0.5967 & 0.6087 & AUC & 0.87168 & 0.87265 & 0.7727 & 0.6818 & 0.635 & 0.5909 \\
\hline $\begin{array}{l}\text { Segment } \\
\text { (BRICKFACE- } \\
\text { others) }\end{array}$ & & & & & & & $\begin{array}{l}\text { Abalone } \\
(18-9)\end{array}$ & & & & & & \\
\hline Accuracy & 0.98095 & 0.99177 & 0.9931 & 0.9965 & 0.9948 & 0.9965 & Accuracy & 0.79891 & 0.84131 & 0.9451 & 0.9451 & 0.9451 & 0.9451 \\
\hline BACC & 0.97879 & 0.98636 & 0.9955 & 0.9958 & 0.9819 & 0.988 & BACC & 0.77035 & 0.78167 & 0.9451 & 0.9451 & 0.5 & 0.5 \\
\hline G-mean & 0.97878 & 0.98633 & 0.9808 & 0.9879 & 0.9818 & 0.9879 & G-mean & 0.76968 & 0.77876 & 0 & 0 & 0 & 0 \\
\hline AUC & 0.99717 & 0.99792 & 0.9809 & 0.988 & 0.9819 & 0.988 & AUC & 0.80406 & 0.83779 & 0.5 & 0.5 & 0.5 & 0.5 \\
\hline $\begin{array}{c}\text { Libra } \\
\text { (123-others) }\end{array}$ & & & & & & & $\begin{array}{c}\text { Vowel } \\
\text { (1-others) }\end{array}$ & & & & & & \\
\hline Accuracy & 0.875 & 0.81389 & 0.9667 & 0.9778 & 0.9778 & 0.9667 & Accuracy & 0.8428 & 0.83333 & 0.8939 & 0.9167 & 0.8712 & 0.8864 \\
\hline BACC & 0.82813 & 0.81597 & 0.9644 & 0.9689 & 0.9444 & 0.9167 & BACC & 0.85729 & 0.87083 & 0.8712 & 0.8848 & 0.9292 & 0.9375 \\
\hline G-mean & 0.82443 & 0.81596 & 0.9129 & 0.9428 & 0.9428 & 0.9129 & G-mean & 0.85711 & 0.86963 & 0.9399 & 0.9531 & 0.9265 & 0.9354 \\
\hline AUC & 0.79352 & 0.84245 & 0.9167 & 0.9444 & 0.9444 & 0.9167 & AUC & 0.90464 & 0.92214 & 0.9417 & 0.9542 & 0.9292 & 0.9375 \\
\hline
\end{tabular}


Table 18. Cont.

\begin{tabular}{|c|c|c|c|c|c|c|c|c|c|c|c|c|c|}
\hline Dataset & SMOTE & $\begin{array}{l}\text { ACOR- } \\
\text { SMOTE }\end{array}$ & $\begin{array}{c}\text { ALO- } \\
\text { SMOTE }\end{array}$ & $\begin{array}{l}\text { MALO- } \\
\text { SMOTE }\end{array}$ & $\begin{array}{c}\text { GWO- } \\
\text { SMOTE }\end{array}$ & $\begin{array}{l}\text { WOA- } \\
\text { SMOTE }\end{array}$ & Dataset & SMOTE & $\begin{array}{l}\text { ACOR- } \\
\text { SMOTE }\end{array}$ & $\begin{array}{c}\text { ALO- } \\
\text { SMOTE }\end{array}$ & $\begin{array}{l}\text { MALO- } \\
\text { SMOTE }\end{array}$ & $\begin{array}{c}\text { GWO- } \\
\text { SMOTE }\end{array}$ & $\begin{array}{l}\text { WOA- } \\
\text { SMOTE }\end{array}$ \\
\hline $\begin{array}{l}\text { Libra (456- } \\
\text { others) }\end{array}$ & & & & & & & $\begin{array}{c}\text { Vowel } \\
\text { (2-others) }\end{array}$ & & & & & & \\
\hline BACC & 0.90972 & 0.91319 & 0.9711 & 0.98 & 0.9444 & 0.9722 & BACC & 0.86563 & 0.87604 & 0.9485 & 0.9379 & 0.9625 & 0.9667 \\
\hline G-mean & 0.90906 & 0.9124 & 0.9428 & 0.9718 & 0.9428 & 0.9718 & G-mean & 0.8651 & 0.8751 & 0.9704 & 0.9747 & 0.9618 & 0.9661 \\
\hline AUC & 0.97796 & 0.97666 & 0.9444 & 0.9722 & 0.9444 & 0.9722 & AUC & 0.9191 & 0.92374 & 0.9708 & 0.975 & 0.9625 & 0.9667 \\
\hline $\begin{array}{l}\text { Vehicle } \\
\text { (van- } \\
\text { others) }\end{array}$ & & & & & & & $\begin{array}{c}\text { Vowel } \\
\text { (3-others) }\end{array}$ & & & & & & \\
\hline Accuracy & 0.95035 & 0.94563 & 0.9573 & 0.9573 & 0.9573 & 0.9573 & Accuracy & 0.85417 & 0.82765 & 0.9848 & 0.9924 & 0.9848 & 0.9924 \\
\hline BACC & 0.95536 & 0.93488 & 0.9488 & 0.9507 & 0.9238 & 0.9238 & BACC & 0.82604 & 0.83958 & 0.9848 & 0.9924 & 0.9167 & 0.9583 \\
\hline G-mean & 0.95532 & 0.93465 & 0.9293 & 0.9293 & 0.9216 & 0.9216 & G-mean & 0.82533 & 0.83946 & 0.9129 & 0.9574 & 0.9129 & 0.9574 \\
\hline $\begin{array}{l}\text { AUC } \\
\text { Glass }\end{array}$ & 0.98235 & 0.98442 & 0.9307 & 0.9307 & 0.9238 & 0.9238 & AUC & 0.9125 & 0.92925 & 0.9167 & 0.9583 & 0.9167 & 0.9583 \\
\hline $\begin{array}{c}\text { (tableware- } \\
\text { others) }\end{array}$ & & & & & & & German & & & & & & \\
\hline Accuracy & 0.82243 & 0.98598 & 0.9811 & 0.9811 & 0.9623 & 0.9623 & Accuracy & 0.731 & 0.729 & 0.716 & 0.716 & 0.716 & 0.708 \\
\hline AUC & 0.90515 & 0.99675 & 0.75 & 0.75 & 0.7402 & 0.7402 & AUC & 0.7891 & 0.79457 & 0.5648 & 0.5648 & 0.5571 & 0.5438 \\
\hline
\end{tabular}




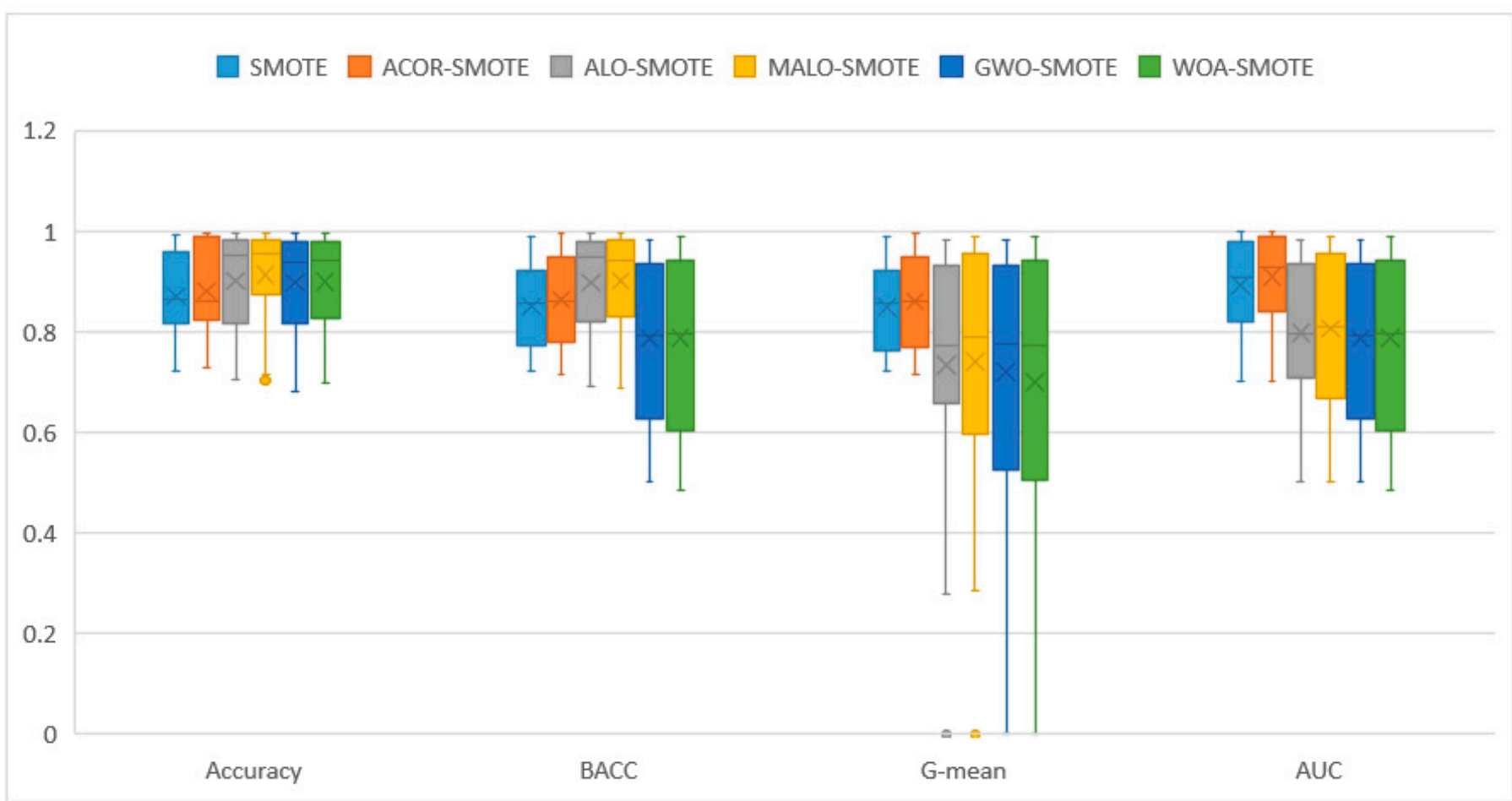

Figure 7. Box and whiskers plots for Accuracy, BACC, G-mean, and AUC of compared methods.

\section{Conclusions}

An optimization-based method was proposed in this paper for the problem of instance reduction to obtain better results in terms of many metrics in both balanced and imbalanced data. A new modified antlion optimization (MALO) method was adapted for this task after validating its ability in terms of optimization compared to state-of-the-art optimizers using benchmark functions. The results obtained at 500 and 1000 iterations for twenty-three and thirteen benchmark functions, respectively, demonstrated that the proposed MALO algorithm could escape the local optima and provide a better convergence rate as compared to the basic ALO algorithm and state-of-the-art optimizers.

Additionally, instance reduction results from MALO were compared to basic antlion Optimization and some well-known optimization algorithms on 15 balanced and imbalanced datasets to test the performance on reducing instances of the training data. Furthermore, antlion optimization and MALO were used to perform training data reduction for 18 oversampled imbalanced datasets, and the reduced datasets were classified by SVM in all experiments. The results were also compared with one novel resampling method.

Obtained results demonstrated that the proposed MALO was superior in minimizing the number of training set instances, hence maximizing the classification performance while reducing the run time compared to state-of-the-art methods used to reduce the original balanced and imbalanced datasets without the need to perform oversampling pre-processing methods which consume computational time and memory space. MALO reduced the instances of oversampled imbalanced datasets with better performance compared to the full oversampled dataset and the recently proposed ACOR instance reduction method, ALO, GWO, and WOA.

The MALO algorithm results showed an increment in Accuracy, BACC, G-mean, and AUC rates up to $7 \%, 3 \%, 15 \%$, and $9 \%$, respectively, for some datasets over the basic ALO algorithm while keeping less computational time.

The need for determining the best values of the parameters of MALO can seem to be a limitation; however, the instance reduction problem in balanced and imbalanced data is a complex problem that can be encountered in many real-world applications and this limitation can be resolved by adjusting the parameters by adopting different statistical concepts. 
Owing to the encouraging outcomes and high performance of MALO in the instance reduction challenge, numerous new evolutionary optimization algorithms can be adjusted for improved outcomes for this hot research area. Multi-objective or many-objective versions of the evolutionary optimization methods can also be adapted to obtain a wider range of non-dominating and alternative solutions that can promote more appropriate roles for this important task by instantaneously satisfying the different conflicting and contradictory objectives using only a single optimization routine.

Current or new evolutionary optimization and search methods can also be hybridized for performance increment by employing the two or more combined methods and eliminating the possible disadvantages of the methods. In this way, a good balance between exploration and exploitation can enhance the performance of the proposed MALO, for instance, the reduction problem in balanced and imbalanced data.

Different techniques for decreasing the computational cost can be embedded in the proposed MALO. Adaptive versions of the methods can also be proposed. Different initialization methods can be integrated into MALO in order to obtain better results for instance reduction problems and other complex real-world problems by obtaining a more uniform population. As another future direction of work, the proposed model can be adapted for real big data classification processes.

Author Contributions: Conceptualization, All authors; methodology, L.M.E.B. and A.S.D.; software, L.M.E.B. and A.S.D.; validation, All authors; formal analysis, L.M.E.B. and A.S.D.; investigation, S.H.; resources, M.A.I.; data curation, B.A.; writing-original draft preparation, L.M.E.B., A.S.D., S.H., B.A. and M.A.I.; writing-review and editing, B.A.; visualization, L.M.E.B., A.S.D., M.A.C. and S.K., A.S; supervision, L.M.E.B., A.S.D., B.A., S.H. and M.A.I.; project administration, L.M.E.B. and A.S.D.; funding acquisition, M.A.C. and S.K. All authors have read and agreed to the published version of the manuscript.

Funding: This research received no external funding.

Institutional Review Board Statement: Not applicable.

Data Availability Statement: Data available at: https://archive.ics.uci.edu/.

Conflicts of Interest: The authors declare no conflict of interest.

\section{References}

1. Abdar, M.; Nasarian, E.; Zhou, X.; Bargshady, G.; Wijayaningrum, V.N.; Hussain, S. Performance Improvement of Decision Trees for Diagnosis of Coronary Artery Disease Using Multi Filtering Approach. In Proceedings of the 2019 IEEE 4th International Conference on Computer and Communication Systems (ICCCS), Singapore, 23-25 February 2019; Institute of Electrical and Electronics Engineers (IEEE): Manhattan, NY, USA, 2019; pp. 26-30.

2. Shoeibi, A.; Ghassemi, N.; Khodatars, M.; Jafari, M.; Hussain, S.; Alizadehsani, R.; Acharya, U.R. Epileptic seizure detection using deep learning techniques: A Review. Int. J. Environ. Res. Public Health 2021, 18, 5780. [CrossRef] [PubMed]

3. Khodatars, M.; Shoeibi, A.; Sadeghi, D.; Ghaasemi, N.; Jafari, M.; Moridian, P.; Khadem, A.; Alizadehsani, R.; Zare, A.; Kong, Y.; et al. Deep learning for neuroimaging-based diagnosis and rehabilitation of Autism Spectrum Disorder: A review. Comput. Biol. Med. 2021, 139, 104949. [CrossRef] [PubMed]

4. Alizadehsani, R.; Sani, Z.A.; Behjati, M.; Roshanzamir, Z.; Hussain, S.; Abedini, N.; Hasanzadeh, F.; Khosravi, A.; Shoeibi, A.; Roshanzamir, M.; et al. Risk factors prediction, clinical outcomes, and mortality in COVID-19 patients. J. Med. Virol. 2021, 93, 2307-2320. [CrossRef] [PubMed]

5. Abdar, M.; Pourpanah, F.; Hussain, S.; Rezazadegan, D.; Liu, L.; Ghavamzadeh, M.; Fieguth, P.; Cao, X.; Khosravi, A.; Acharya, U.R.; et al. A review of uncertainty quantification in deep learning: Techniques, applications and challenges. Inf. Fusion 2021, 76, 243-297. [CrossRef]

6. Koohestani, A.; Abdar, M.; Hussain, S.; Khosravi, A.; Nahavandi, D.; Nahavandi, S.; Alizadehsani, R. Analysis of Driver Performance Using Hybrid of Weighted Ensemble Learning Technique and Evolutionary Algorithms. Arab. J. Sci. Eng. 2021, 46, 3567-3580. [CrossRef]

7. Hussain, S.; Hazarika, G. Educational Data Mining Model Using Rattle. Int. J. Adv. Comput. Sci. Appl. 2014, 5. [CrossRef]

8. Basiri, M.E.; Nemati, S.; Abdar, M.; Cambria, E.; Acharrya, U.R. ABCDM: An Attention-based Bidirectional CNN-RNN Deep Model for sentiment analysis. Futur. Gener. Comput. Syst. 2021, 115, 279-294. [CrossRef]

9. Desuky, A.S.; Hussain, S. An Improved Hybrid Approach for Handling Class Imbalance Problem. Arab. J. Sci. Eng. 2021, 46, 3853-3864. [CrossRef] 
10. Chou, J.-S.; Truong, D.-N. A novel metaheuristic optimizer inspired by behavior of jellyfish in ocean. Appl. Math. Comput. 2021, 389, 125535. [CrossRef]

11. Negi, G.; Kumar, A.; Pant, S.; Ram, M. Optimization of Complex System Reliability using Hybrid Grey Wolf Optimizer. Decis. Mak. Appl. Manag. Eng. 2021, 4, 241-256. [CrossRef]

12. Das, M.; Roy, A.; Maity, S.; Kar, S.; Sengupta, S. Solving fuzzy dynamic ship routing and scheduling problem through new genetic algorithm. Decis. Mak. Appl. Manag. Eng. 2021. [CrossRef]

13. Ganguly, S. Multi-objective distributed generation penetration planning with load model using particle swarm optimization. Decis. Mak. Appl. Manag. Eng. 2020, 3, 30-42. [CrossRef]

14. Carvajal, O.; Melin, P.; Miramontes, I.; Prado-Arechiga, G. Optimal design of a general type-2 fuzzy classifier for the pulse level and its hardware implementation. Eng. Appl. Artif. Intell. 2021, 97, 104069. [CrossRef]

15. Precup, R.-E.; David, R.-C.; Roman, R.-C.; Petriu, E.M.; Szedlak-Stinean, A.-I. Slime Mould Algorithm-Based Tuning of CostEffective Fuzzy Controllers for Servo Systems. Int. J. Comput. Intell. Syst. 2021, 14, 1042-1052. [CrossRef]

16. Valdez, F.; Castillo, O.; Cortes-Antonio, P.; Melin, P. A survey of Type-2 fuzzy logic controller design using nature inspired optimization. J. Intell. Fuzzy Syst. 2020, 39, 6169-6179. [CrossRef]

17. Hu, H.; Wang, H.; Bai, Y.; Liu, M. Determination of endometrial carcinoma with gene expression based on optimized Elman neural network. Appl. Math. Comput. 2019, 341, 204-214. [CrossRef]

18. Gupta, N.; Jain, R.; Gupta, D.; Khanna, A.; Khamparia, A. Modified Ant Lion Optimization Algorithm for Improved Diagnosis of Thyroid Disease. In Advances in Human Error, Reliability, Resilience, and Performance; Springer Science and Business Media LLC: Singapore, 2020; pp. 599-610.

19. El-Kenawy ES, M.; Eid, M.M.; Saber, M.; Ibrahim, A. MbGWO-SFS: Modified binary grey wolf optimizer based on stochastic fractal search for feature selection. IEEE Access 2020, 8, 107635-107649. [CrossRef]

20. Lin, K.-C.; Zhang, K.-Y.; Huang, Y.-H.; Hung, J.C.; Yen, N.Y. Feature selection based on an improved cat swarm optimization algorithm for big data classification. J. Supercomput. 2016, 72, 3210-3221. [CrossRef]

21. Wan, Y.; Wang, M.; Ye, Z.; Lai, X. A feature selection method based on modified binary coded ant colony optimization algorithm. Appl. Soft Comput. 2016, 49, 248-258. [CrossRef]

22. Zakeri, A.; Hokmabadi, A. Efficient feature selection method using real-valued grasshopper optimization algorithm. Expert Syst. Appl. 2019, 119, 61-72. [CrossRef]

23. Nanni, L.; Lumini, A. Particle swarm optimization for prototype reduction. Neurocomputing 2009, 72, 1092-1097. [CrossRef]

24. Zhai, T.; He, Z. Instance selection for time series classification based on immune binary particle swarm optimization. Knowl. Based Syst. 2013, 49, 106-115. [CrossRef]

25. Hamidzadeh, J.; Monsefi, R.; Yazdi, H.S. LMIRA: Large Margin Instance Reduction Algorithm. Neurocomputing 2014, 145 , 477-487. [CrossRef]

26. Saidi, M.; Bechar, M.E.A.; Settouti, N.; Chikh, M.A. Instances selection algorithm by ensemble margin. J. Exp. Theor. Artif. Intell. 2017, 30, 457-478. [CrossRef]

27. Carbonera, J.L.; Abel, M. A Density-Based Approach for Instance Selection. In Proceedings of the 2015 IEEE 27 th International Conference on Tools with Artificial Intelligence (ICTAI), Vietri sul Mare, Italy, 9-11 November 2015; Institute of Electrical and Electronics Engineers (IEEE): Manhattan, NY, USA, 2015; pp. 768-774.

28. De Haro-García, A.; Cerruela-García, G.; García-Pedrajas, N. Instance selection based on boosting for in-stance-based learners. Pattern Recognit. 2019, 96, 106959. [CrossRef]

29. Wang, M.; Heidari, A.A.; Chen, M.; Chen, H.; Zhao, X.; Cai, X. Exploratory differential ant lion-based optimization. Expert Syst. Appl. 2020, 159, 113548. [CrossRef]

30. Pierezan, J.; Coelho, L.d.S.; Mariani, V.C.; Goudos, S.K.; Boursianis, A.D.; Kantartzis, N.V.; Antonopoulos, C.S.; Nikolaidis, S. Multiobjective Ant Lion Approaches Applied to Electromagnetic Device Optimization. Technologies 2021, 9, 35. [CrossRef]

31. Assiri, A.S.; Hussien, A.G.; Amin, M. Ant Lion Optimization: Variants, Hybrids, and Applications. IEEE Access 2020, 8, 77746-77764. [CrossRef]

32. Tian, T.; Liu, C.; Guo, Q.; Yuan, Y.; Li, W.; Yan, Q. An improved ant lion optimization algorithm and its application in hydraulic turbine governing system parameter identification. Energies 2018, 11, 95. [CrossRef]

33. Mirjalili, S. The antlion optimizer. Adv. Eng. Softw. 2015, 83, 80-98. [CrossRef]

34. Chawla, N.V.; Bowyer, K.W.; Hall, L.O.; Kegelmeyer, W.P. SMOTE: Synthetic minority over-sampling technique. J. Artif. Intell. Res. 2002, 16, 321-357. [CrossRef]

35. Mirjalili, S.; Mirjalili, S.M.; Lewis, A. Grey Wolf Optimizer. Adv. Eng. Softw. 2014, 69, 46-61. [CrossRef]

36. Mirjalili, S.; Lewis, A. The whale optimization algorithm. Adv. Eng. Softw. 2016, 95, 51-67. [CrossRef]

37. Wang, L. Support Vector Machines: Theory and Applications; Springer: Berlin/Heidelberg, Germany, 2005.

38. Rashedi, E.; Nezamabadi-Pour, H.; Saryazdi, S. GSA: A Gravitational Search Algorithm. Inf. Sci. 2009, 179, 2232-2248. [CrossRef]

39. Kennedy, J.; Eberhart, R. Particle swarm optimization. In Proceedings of the ICNN'95-International Conference on Neural Networks, Perth, Australia, 27 November-1 December 1995; Volume 4, pp. 1942-1948.

40. Hashim, F.A.; Hussain, K.; Houssein, E.H.; Mabrouk, M.S.; Al-Atabany, W. Archimedes optimization algorithm: A new metaheuristic algorithm for solving optimization problems. Appl. Intell. 2021, 51, 1531-1551. [CrossRef] 
41. Yang, X.-S. A New Metaheuristic Bat-Inspired Algorithm. In Nature Inspired Cooperative Strategies for Optimization (NICSO 2010); González, J.R., Pelta, D.A., Cruz, C., Terrazas, G., Krasnogor, N., Eds.; Studies in Computational Intelligence; Springer: Berlin/Heidelberg, Germany, 2010; pp. 65-74.

42. Cuevas, E.; Echavarría, A.; Ortegón, M.A.R. An optimization algorithm inspired by the States of Matter that improves the balance between exploration and exploitation. Appl. Intell. 2014, 40, 256-272. [CrossRef]

43. Cuevas, E.; Echavarría, A.; Zaldívar, D.; Pérez-Cisneros, M. A novel evolutionary algorithm inspired by the states of matter for template matching. Expert Syst. Appl. 2013, 40, 6359-6373. [CrossRef]

44. Yang, X.-S. Flower Pollination Algorithm for Global Optimization. In Proceedings of the Image Analysis and Processing-ICIAP 2017, Orléan, France, 3-7 September 2012; Springer Science and Business Media LLC: Berlin/Heidelberg, Germany, 2012; pp. 240-249.

45. Holland, J.H. Genetic algorithms. Sci. Am. 1992, 267, 66-73. [CrossRef]

46. Yang, X.-S.; Algorithm, F.; Flights, L.; Optimization, G. Research and Development in Intelligent Systems XXVI; Springer Science and Business Media LLC: Berlin/Heidelberg, Germany, 2009; pp. 209-218.

47. Yang, X.-S. Firefly algorithm, stochastic test functions and design optimisation. Int. J. Bio-Inspired Comput. 2010, 2, 78. [CrossRef]

48. Yang, X.S.; Deb, S. Cuckoo search via Lévy flights. In Proceedings of the 2009 World Congress on Nature \& Biologically Inspired Computing (NaBIC), Coimbatore, India, 9-11 December 2009; pp. 210-214.

49. Asuncion, A.; Newman, D. UCI Machine Learning Repository. 2007. Available online: https://archive.ics.uci.edu/ (accessed on 5 February 2022).

50. Sheskin, D.J. Handbook of Parametric and Nonparametric Statistical Procedures; CRC Press: Boca Raton, FL, USA, 2003; Volume 51, p. 374; ISBN 1420036262.

51. Li, M.; Xiong, A.; Wang, L.; Deng, S.; Ye, J. ACO Resampling: Enhancing the performance of oversampling methods for class imbalance classification. Knowl. Based Syst. 2020, 196, 105818. [CrossRef]

52. Han, H.; Wang, W.Y.; Mao, B.H. Borderline-SMOTE: A new over-sampling method in imbalanced data sets learning. In International Conference on Intelligent Computing; Springer: Berlin/Heidelberg, Germany, 2005; pp. 878-887.

53. He, H.; Bai, Y.; Garcia, E.A.; Li, S. ADASYN: Adaptive synthetic sampling approach for imbalanced learning. In Proceedings of the IEEE International Joint Conference on Neural Networks, Hong Kong, China, 1-8 June 2008; pp. 1322-1328. [CrossRef]

54. Mease, D.; Wyner, A.J.; Buja, A. Boosted classification trees and class probability/quantile estimation. J. Mach. Learn. Res. 2007, $8,409-439$.

55. Colorni, A.; Dorigo, M.; Maniezzo, V. Distributed optimization by ant colonies. In Proceedings of the First European Conference on Artificial Life (ECAL'91), Paris, France, 11-13 December 1991; Elsevier Publishing: Amsterdam, The Netherlands, 1991; Volume 142, pp. 134-142.

56. Youn, E.; Jeong, M.K. Class dependent feature scaling method using naive Bayes classifier for text datamining. Pattern Recognit. Lett. 2009, 30, 477-485. [CrossRef]

57. Quinlan, J.R. C4. 5: Programs for Machine Learning; Elsevier: Amsterdam, The Netherlands, 2014.

58. Cristianini, N.; Shawe-Taylor, J. An Introduction to Support Vector Machines and Other Kernel-Based Learning Methods; Cambridge University Press: Cambridge, UK, 2000. 